\title{
1 Decoding the effects of synonymous variants
}

2 Zishuo Zeng ${ }^{1 *}$, Ariel A. Aptekmann ${ }^{1}$, and Yana Bromberg ${ }^{1,2^{*}}$

31 Department of Biochemistry and Microbiology, Rutgers University, New Brunswick, NJ 08873, USA

42 Department of Genetics, Rutgers University, Piscataway, New Jersey 08854, USA

5 * Corresponding author:zzeng@bromberglab.org, yana@bromberglab.org

6 Tel: +1-732-932-9763 Ext. 218; Fax +1-732-932-8965

\section{ABSTRACT}

9 Synonymous single nucleotide variants (sSNVs) are common in the human genome but are often 10 overlooked. However, sSNVs can have significant biological impact and may lead to disease. Existing 11 computational methods for evaluating the effect of sSNVs suffer from the lack of gold-standard 12 training/evaluation data and exhibit over-reliance on sequence conservation signals. We developed 13 synVep (synonymous Variant effect predictor), a machine learning-based method that overcomes both of 14 these limitations. Our training data was a combination of variants reported by gnomAD (observed) and 15 those unreported, but possible in the human genome (generated). We used positive-unlabeled learning to 16 purify the generated variant set of any likely unobservable variants. We then trained two sequential 17 extreme gradient boosting models to identify subsets of the remaining variants putatively enriched and 18 depleted in effect. Our method attained $90 \%$ precision/recall on a previously unseen set of variants.

19 Furthermore, although synVep does not explicitly use conservation, its scores correlated with evolutionary

20 distances between orthologs in cross-species variation analysis. synVep was also able to differentiate 21 pathogenic vs. benign variants, as well as splice-site disrupting variants (SDV) vs. non-SDVs. Thus, 22 synVep provides an important improvement in annotation of SSNVs, allowing users to focus on variants 23 that most likely harbor effects.

\section{INTRODUCTION}

26 The recent increase in accessibility of sequencing has facilitated a rise in precision medicine efforts 27 focused on the interpretation of the effects of individual-specific genome variation (1). Genome-wide 28 association studies (GWAS) have identified multiple variants marking specific phenotypes (2). However, 29 the evaluation of variants in terms of their functional contributions to molecular pathogenicity mechanisms

30 holds promise for both a better understanding of disease and drug discovery/optimization (3). SNVs 31 (single nucleotide variants) are the most common variants in the human genome (4). Three types of 32 SNVs are of particular interest - regulatory (i.e. changing the quantity/production of the gene product, e.g. 33 transcription or splice site variants), non-synonymous (i.e. altering product protein sequence), and 34 synonymous (i.e. variants in protein-coding regions that, due to the degeneracy of the genetic code, do 35 not alter the protein sequence). Many computational tools have been developed to evaluate the functional 36 effects of regulatory and non-synonymous variants $(5,6)$. However, while an individual genome carries as 
37 many synonymous as non-synonymous SNVs (7), the former are often disregarded as functionally irrelevant. Still, sSNVs can cause disease (8) and affect gene function via multiple mechanisms, including

39 binding of transcription factors (9), splicing (10), mRNA stability (11-13), co-translational folding (14-16),

40 etc., as reviewed in our earlier work (17).

41 Existing methods for predicting sSNV effects are either (1) sSNV-specific tools, including SilVA (18), reg42 SNP-splicing (19), DDIG-SN (20), TraP (21), and IDSV (22), or (2) general-purpose ones, including 43 CADD $(23,24)$, DANN (25), FATHMM-MKL (26), and MutationTaster2 (27). The number of computational 44 sSNV effect predictors is limited in comparison to that of nsSNV (non-synonymous single nucleotide 45 variant) effect predictors, as reviewed in $(6,17)$. Partially, this paucity is due to the limited available 46 experimental data evaluating variant effects, which could be used for training or testing of such methods. 47 In fact, all existing predictors, except CADD and DANN, are trained using "pathogenic" variants from 48 databases such as Human Gene Mutation Database (HGMD) (28) and ClinVar (29). Here we note that 49 "pathogenicity" is not equivalent to "functional effect" $(30,31)$ and inferring variant-disease causality is 50 complicated by this inequality. The experimental disease variant annotations are also often unreliable 51 (17), as it is difficult to distinguish causative variants from simply associated ones. Moreover, the 52 pathogenic label is inconsistent across databases, and possibly over time/database releases. Finally, 53 even these labeled effect variants are few; even fewer are experimentally labeled neutral polymorphisms. 54 Thus, predictors trained on these variants are likely insufficient to predict the effects of tens of millions of 55 possible sSNVs in human genome.

56 Using positive-unlabeled learning (32-34), we inferred a subset of human sSNVs that could be used for 57 training a predictor of sSNV molecular effect. We then developed, synVep (synonymous Variant Effect 58 Predictor), a machine learning-based method for scoring putative effect for each possible human sSNV. 59 synVep discriminated experimentally validated pathogenic sSNVs from randomly sampled common 60 variants. Its predictions also displayed the expected trends (35) in evolutionary distances between 61 orthologs, where the sSNVs corresponding to evolutionarily-close human relatives' (e.g. chimp) reference 62 nucleotide, have lower effect scores than those corresponding to the nucleotides of further away 63 organisms (e.g. fruitfly). Furthermore, nucleotides that are not identified in any of the species evaluated 64 here are deemed to have most effect when substituted into the human reference. However, many of the 65 sSNVs that are not observed in the human population, tend to be scored very high (most effect), 66 regardless of their appearance in other species.

67 In line with our earlier observations (17), we find that the variant frequency in the population is poorly 68 correlated with the effect score; i.e rare variants are about equally likely to have no effect on gene 69 function as common variants (65\% common vs $69 \%$ rare). synVep does not rely on conservation and is 70 developed without an experimental or explicitly evolutionarily estimated gold standard 71 training/development set. Its success thus suggests the feasibility of a similar approach for the 72 development of a training set for other variant types, e.g. nsSNVs or indels. We expect that synVep sSNV 
73 effect predictions will greatly contribute to our understanding of pathogenicity pathways and to the

74 prioritization of synonymous variants in disease.

\section{MATERIALS AND METHODS}

76 Data Collection. We extracted all 93,437 human protein-coding transcripts from the Ensembl BioMart (36) GRCh37 p.13 assembly (37) and discarded the ones containing unknown nucleotides, lacking a start/stop codon, or having patched (https://grch37.ensembl.org/Homo sapiens/Info/Annotation) chromosome IDs. We then generated all possible sSNVs for the remaining 72,400 transcripts. We further used ANNOVAR (38) (installed Aug 5, 2019) to extract sSNVs in these transcripts, and their allele-count based frequencies, from the Genome Aggregation Database exome subset (gnomAD exome) (39). An

82 sSNV present in gnomAD was labeled a singleton if it was seen in only one individual and otherwise

83 labeled observed. Generated sSNVs were those in the set of all possible variants in the 72,400 84 transcripts that were not singleton or observed. Thus, we collected 4,160,063 observed, 3,438,470 85 singleton, and 57,208,450 generated sSNVs (https://zenodo.org/record/4763256). Note that these 86 correspond to 1,520,334 observed, 1,233,878 singleton, and 21,314,668 generated sSNVs with unique 87 genomic coordinates and reference/alternative alleles, i.e. in one transcript per gene.

88 To evaluate and compare the performance of our predictor to other predictors, we manually curated a 89 dataset of 42 curated-effect sSNVs with known biological effects, including the 33 pathogenic variants 90 from the Buske et al. study (18). We required that all sSNVs in this set were strongly associated with

91 disease and that there was experimental evidence of their molecular effects. These 42 sSNVs 92 (Supplementary Table S1) mapped to 170 transcript-based sSNVs and were excluded from model 93 training throughout this manuscript.

94 Variant Features. We collected 35 variant and sequence features (Supplementary Table S2), grouped into six categories: codon bias and autocorrelation (10 features), protein structure (3), mRNA stability (8), distance to regulatory factors (4), expression profile (3), and miscellaneous (7). The reasons for selecting these features are described in our earlier paper (17). We further calculated the correlation of feature values across all sSNVs using the dython package (v0.6.7, https://github.com/shakedzy/dython), where correlations between continuous-continuous, continuous-categorical, and categorical-categorical features 100 were computed using Pearson correlation (40), Cramer's V (41), and correlation ratio (42), respectively.

101 Feature importance was obtained by calculating the average performance gain across all splits where the 102 feature was present.

103 1. Transcript expression profiles. We downloaded the GTEx (43) "Transcript TPMs" dataset (dbGaP 104 Accession phs000424.v7.p2) and standardized the transcript expression across tissue samples. We then 105 used the average expression of each transcript over all samples from the same tissue as the 106 representative transcript expression for that tissue.

107 Calculations of some of the codon bias metrics described below require a reference set of coding 108 sequences, which are typically a set of highest expressed transcripts (44). To identify these references, 
109 we collected the maximum expression values for all transcripts across the 53 tissues. We then selected 110 the transcripts within the highest $1 \%$ expression per tissue. We also used $\log _{10}$ (minimum expression per 111 tissue), $\log _{10}$ (median expression per tissue), and $\log _{10}$ (maximum expression per tissue) for each 112 transcript as features.

113 2. Codon bias and autocorrelation. A variety of measures and/or their " $\Delta$ " form (difference in measure 114 value after mutation vs. value before mutation) are adopted as features to characterize the codon bias of 115 transcripts (see Supplementary text for more details), including the Codon Adaptation Index (CAI, 116 Supplementary text Eqn. 1) (44), Fraction of Optimal Codons (fracOpt, Supplementary text Eqn. 2) (45), 117 Codon Usage Bias (CUB, Supplementary text Eqn. 3) (46), Intrinsic Codon Deviation Index (ICDI, 118 Supplementary text Eqn. 4) (47), Synonymous Codon Usage Order (SCUO, Supplementary text Eqn. 5) 119 (48), and tRNA Adaptation Index (tAI, Supplementary Eqn. 6) (49). The calculation of these values was 120 performed in $\mathrm{R}$ (50) and is available as an $\mathrm{R}$ package in 121 https://bitbucket.org/bromberglab/codonbiasmetrics/src/master/.

122 These measures describe codon bias from different perspectives. CAI, fracOpt, and CUB rely on a 123 reference set of optimal codons, found in highly expressed genes (51). CAl computes the geometric 124 mean of relative usage of a codon compared to the most frequently used codon for the same amino acid 125 (44). fracOpt is the fraction of optimal codons in a sequence of a certain length. CUB weighs the 126 frequency of amino acids in calculating codon bias. ICDI is independent of a reference set of genes (47). 127 SCUO borrows the idea of entropy from Shannon information theory to describe codon usage bias of 128 sequences (48). tAl focuses on translational efficiency by taking tRNA levels into account (49).

129 We also considered codon autocorrelation - a feature that has not yet been used by any sSNV 130 predictors. In autocorrelated sequences same codons cluster together, whereas they are separated in 131 anticorrelated sequences (e.g. XXXYYY is more autocorrelated than XYXYXY, where $X$ and $Y$ are two 132 different codons) (52). Cannarozzi et al. noted the association between codon autocorrelation and 133 translation dynamics and proposed the tRNA pairing index (TPI) to describe a sequence's codon 134 autocorrelation. Autocorrelated sequences benefit from rapid translation due to the recycling of 135 isoaccepting tRNAs (52). However, we note that the significance of recycling is likely weaker if the interval 136 between two issoaccepting codons is larger - a feature that is not accounted for in TPI. Therefore, we 137 proposed a new measure, Codon Autocorrelation Measure (CAM, Supplementary Eqn. 7), to describe the 138 variant-specific codon autocorrelation impact penalized by the distance between the synonymous codons.

139 Finally, we also introduced the change of frequency measure (CF, Supplementary text Eqn. 8), to 140 describe the amount of impact on codon's frequency in a sequence due to the introduction of the variant.

1413 . Distance to regulatory and splicing sites. We used as features the distances to the nearest splice sites, 142 transcription factor binding site (TFBS), RNA-binding protein (RBP) motif, and exonic splicing regulator 143 (ESR). Their genomic coordinates were obtained from different sources as described below. We then 144 computed the distance of a variant (in nucleotides) to all regulatory sites and selected the minimum value 
145 as the feature distance. We categorized these distances $(d)$ into six categories as feature inputs: $d=0$, $1460<d<=3,3<d<=5,5<d<=10,10<d<=20$, and $d>20$.

147 Genomic coordinates of regulatory regions were inferred as follows: (1) Splice sites were inferred from the 148 "Genomic coding start" and "Genomic coding end" of all human protein-coding transcripts annotated in 149 Ensembl BioMart GRCh37 p.13 assembly. (2) We downloaded the Gene Transcription Regulation 150 Database (GTRD, version 18.06) (53) and identified the genomic coordinates of TFBS, using hg38 to 151 hg19 conversion via CrossMap (54) for correspondence with our transcript coordinates. (3) We 152 downloaded the ATtRACT database of RNA binding proteins and AssoCiated moTifs (55) and mapped 153 the human RPB motifs to our set of transcript sequences. (4) We also downloaded the supplementary 154 data of Cáceres et al (56) gold standard ESR motif set and mapped these to our transcripts.

155 4. Protein structure. We ran PredictProtein (57), a collection of tools for protein structure predictions, on 156 all of the translated transcript sequences. We were particularly interested in protein secondary structure 157 (PSS), residue solvent accessibility (SS), and disorder (PD) predictions; in PredictProtein, PROFphd (58) 158 predicts PSS and SS, while Meta-disorder (MD) (59) predicts PD.

159 5. mRNA stability, structure, and structural changes. We ran RNAfold (60) to predict (with calculation of 160 partition function and base pairing probability matrix) the secondary structure and stability of all 161 transcripts. We extracted the frequency of the structure with minimum free energy (MFE) in the structure 162 ensemble, the free energy of the centroid structure, and its distance to the structure ensemble, as well as 163 the local mRNA structure (strongly paired, strongly up/down -stream paired, weakly paired, weakly 164 up/down -stream paired, or unpaired bases).

165 We also used RNAsnp (61) to predict the variant-induced local secondary structure changes for all 166 sSNVs. The "mode" and "winsizeFold" parameters should be assigned according to the length in 167 nucleotides $(L)$ of the input sequence. We assigned the parameters as follows: $(1)$ for $L<=200, \bmod =1$ 168 and winsizeFold=100; (2) for $200<L<=500$, $\bmod =1$ and winsizeFold=200; (3) for $L>500$, $\bmod =2$ and 169 winsizeFold=500. We recorded the local structure dissimilarity, global structural dissimilarity and their 170 statistical significance ( $p$-values).

171 Model construction Classifier setup. We standardized all continuous features and label-encoded 172 categorical features. We compared two classifiers for differentiating observed and generated variants: 173 deep neural network (DNN) (62) and XGBoost (63); we selected XGBoost as the classification algorithm 174 for its higher accuracy and speed (preliminary experiment described in Supplementary Text, Page 2). 175 XGBoost is implemented in Python (v3.6.4) xgboost package (v0.8.2) integrated with sci-kit learn (0.20.3) 176 (64) (https://xgboost.readthedocs.io/en/latest/python/python api.html).

177 Balancing variant data by transcript. The generated set of SSNVs is much larger than the observed set, 178 but the number of observed sSNVs per transcript varies greatly. Moreover, some classifier input features 179 are transcript-specific. Thus, a predictor may "memorize" transcripts that have more observed sSNVs, 180 and preferentially assign its variants observed status, instead of finding variant-specific differences 
181 between observed and generated. To avoid this, we assigned sampling likelihood weights for the 182 generated set, i.e. the sampling likelihood weight of a generated variant is the number of observed sSNVs 183 in the corresponding transcript. In all further balancing of data sets, generated sSNVs were 184 probabilistically added to the set on the basis of their weights. Thus, the number of generated sSNVs on a 185 transcript that were selected for a particular training set was correlated with the number observed sSNVs 186 on this transcript.

187 Positive unlabeled learning (PUL) to identify unobservable SSNVs. PUL is a semi- supervised approach 188 applicable to scenarios where only positive data points are labeled and the rest can be positive or 189 negative (32-34). We employed the modified version of PUL (34) to separate the generated sSNVs into 190 unobservable and not-seen sets. To prevent overfitting, we adopted relatively conservative 191 hyperparameters of XGBoost (100 trees [n_estimor], 5 maximum depth [max_depth], 30\% of the features 192 per tree [colsample_bytree], 30\% subsamples per tree [subsample]). We left out from PUL a fraction of 193 observed as a test set, aiming to reach $<5 \%$ incorrect predictions for this set at the end of the PUL.

194 In one epoch of PUL, a classifier was trained to differentiate the observed sSNVs from the same number 195 of unlabeled ones (generated; selected via transcript-based set balancing as described above). All 196 unlabeled SSNVs, including the ones not used in training, were evaluated with the resulting model and the 197 generated variants classified as observed (scoring below 0.5) were added to the not-seen pool. The PUL 198 process was repeated until convergence (Supplementary text). sSNVs scoring $>0.5$ in prediction from the 199 last PUL model were further excluded from our data set. One pitfall of this PUL strategy is that a fraction 200 of the unlabeled samples may become positive (observed) with more sequencing in the future.

201 Differentiating the observable from not-seen using an intermediate model. We trained a model to 202 differentiate the observable sSNVs from the not-seen sSNVs (termed intermediate model from here on). 203 We excluded $10 \%(9,274)$ of the common sSNVs (MAF > 0.01; excluded set) and all curated-effect 204 sSNVs (170) from the construction of the intermediate model for testing and final model parameter 205 optimization. We split the observed sSNVs into subsets of 9: 0.5 : 0.5 size ratio for training $(3,631,441$ 206 variants), validation and testing (201,746 variants each). We then randomly sampled the not-seen 207 variants to match the observed validation and test set sizes; this left 47,923,258 not-seen sSNVs for 208 training. We then up-sampled the $3.6 \mathrm{M}$ observed variants in the training set to create a balanced set of $20947,923,258$ observed and not-seen variants, each). We tuned the model hyperparameters by optimizing 210 the F-score (Eqn. 3) of performance on the validation set and evaluated the resulting model on the test 211 set.

$$
\text { Precision }=\frac{T P}{T P+F P}
$$$$
\text { Recall }=\frac{T P}{T P+F N}
$$

214

$$
F_{-} \text {core }=\frac{2 \times \text { Precision } \times \text { Recall }}{(\text { Precision }+ \text { Recall })}
$$

(Eqn. 1)

(Eqn. 2)

(Eqn. 3) 
where TP, TN, FP, FN are respectively, true positive, i.e. observed sSNVs predicted to be observed; true negative, not-seen SSNVs predicted to be not-seen; false positive, not-seen sSNVs predicted to be observed; false negative, observed sSNVs predicted to be not-seen.

Final model (synVep) training. We used the intermediate model to score the excluded common and curated-effect sSNVs, as well as all observed and not-seen sSNVs. Here we assumed that common variants should be enriched in no-effect/neutral variation. Based on the scores of excluded sSNVs, we defined effect and no-effect synVep development sets, where sSNVs (both observed and not-seen) scoring above the median of the curated-effect predictions were deemed effect; while sSNVs (both observed and not-seen) scoring below the median of the excluded common sSNV predictions were labeled no-effect. We thus collected 7,385,137 no-effect and 32,117,625 effect sSNVs.

We split the no-effect and effect sSNVs into subsets of 9: $0.5: 0.5$ size ratio (in the same way as for the intermediate model) for training, validation, and test sets (62,758,222: 735,194: 735,194 variants per set). We sampled equal numbers of effect SSNVs to match the no-effect SSNVs in validation and test sets. We trained the final model on the training set using the hyperparameters optimized (F-score; Eqn. 3) on the validation set. We finally evaluated the model on the test set. Note that none of the curated-effect, the excluded common sSNVs, or the ClinVar (described below) dataset variants were included in our model training.

Performance comparison with other predictors. For all comparisons with other predictors, we calculated the area under the receiver operating characteristic curve (auROC) using the pROC package (65) (v1.17.0.1), and the area under the precision recall curve (auRPC) using PRROC (66) (v1.3.1). Statistical significance of the differences between the auROC/auPRC of synVep and those of other predictors were tested using the pROC package (bootstrap method with the default settings, $n=2,000$; source code was modified to accommodate testing for auPRC).

Common/curated-effect dataset comparison. To evaluate synVep in comparison with other predictors, we used the 170 (transcript-based; 42 genomic coordinate-based) curated-effect sSNVs and the 9,274 (transcript-based; 7,957 genomic coordinate-based) excluded common sSNVs. Here we again assumed that common variants should be enriched in no-effect/neutral variation.

Other predictors in this comparison included: CADD (phred-like scaled scores) (23), DANN (25), FATHMM-MKL (26), DDIG-SN (20), and EIGEN (67). EIGEN scores were collected using ANNOVAR (38) annotations; for other predictors, the scores were collected with default parameters as described in our earlier work (17). We did not include SilVA (18) or TraP (21) in this comparison because 33 of 42 of the curated-effect SSNVs are in their training sets.

Note that synVep scores are produced per variant per transcript, while other predictors use the genomic coordinates, i.e. one reference sequence per variant. For the purposes of our comparison, we randomly re-sampled each tool's predictions of the effect set (42 variant scores) to produce 170 scores. Furthermore, as the common sSNVs (putatively no-effect) outnumbered the effect set, we randomly 
sampled 170 common variant scores in 100 comparison iterations. For each re-sampling, we performed a one-sided permutation test (null hypothesis: mean of common variant scores is equal to mean of effect scores; alternative hypothesis: mean of common variant sores is lower than mean of effect scores) and

254 recorded the $p$-value and the difference of the common and effect variant score distribution medians

255 (Amedians). To assure that all predictor scores fall into the same $[0,1]$ range, we standardized CADD and

256 EIGEN $\triangle$ medians to their maximum non-outlier score (20 for CADD and 3 for EIGEN).

257 We also computed the Spearman correlation across predictor scores and the Fraction of Consensus

258 Binary Predictions (FCBP; i.e. the number of binarized predictions agreed upon by all predictors, divided

259 by total number of predictions) (17). An effect/no-effect scoring threshold for the FCBP computation is 260 required; we used the default value of score $=0.5$ for DANN, FATHMM-MKL, and DDIG-SN. For CADD, 261 we used score=15 as the threshold recommended by its online documentation 262 (https://cadd.gs.washington.edu/info). As there was no recommended cutoff in the EIGEN publication 263 (67), we selected the cutoff (score=1.35) at the 75-percentile of EIGEN scores of 1,000 randomly 264 sampled observed sSNVs.

265 ClinVar dataset comparison. We downloaded all ClinVar (68) submissions from the FTP site 266 (https://ftp.ncbi.nlm.nih.gov/pub/clinvar/) and identified the sSNVs among these. We only considered the 267 sSNVs with the "reviewed by expert panel" review status. From these we selected the (1) pathogenic and 268 pathogenic/likely pathogenic variants as the pathogenic set and (2) benign and benign/likely benign as 269 the benign set. There were 51 benign (genomic coordinate-based; 254 transcript-based) and 17 270 pathogenic (genomic coordinate-based; $n=68$ transcript-based) sSNVs (Supplementary Table S3). We 271 also annotated these ClinVar sSNVs with the precomputed GERP++ scores 272 (http://mendel.stanford.edu/SidowLab/downloads/gerp/) (69).

273 Splicing dataset comparisons. We downloaded and analyzed a dataset of SNV splicing effects (70) 274 (https://github.com/KosuriLab/MFASS), referenced by genomic coordinates and Ensembl transcript IDs. 275 For comparison with synVep, we downloaded and ran spliceAI (71) (https://github.com/llumina/SpliceAl) 276 and retrieved CADD-splice (72) annotation from https://cadd.gs.washington.edu/score. spliceAl 277 predictions are composed of probabilities of splice acceptor and donor's gain and loss. Since these 278 outputs are predominantly zero, we took the maximal value for evaluation purpose, as in (72).

279 Cross-species sequence variation (CSV) analysis. Cross-species variation (CSV) describes the 280 nucleotide difference between the human reference sequence and the ortholog reference sequence of 281 another species. In this study, we selected 20 species to generate CSVs: yeast (Saccharomyces 282 cerevisiae), worm (Caenorhabdiis elegans), fruitfly (Drosophila melanogaster), zebrafish (Danio rerio), 283 xenopus (Xenopus laevis), anole lizard (Anolis carolinensis), chicken (Gallus gallus), platypus 284 (Ornithorhynchus anatinus), opossum (Monodelphis domestica), dog (Canis familiaris), pig (Sus scrofa), 285 dolphin (Tursiops truncatus), mouse (Mus musculus), rabbit (Oryctolagus cuniculus), tree shrew (Tupaia 
belangeri), tarsier (Carlito syrichta), gibbon (Nomascus leucogenys), gorilla (Gorilla gorilla), bonobo (Pan paniscus), and chimpanzee (Pan troglodytes).

288 To represent the evolutionary distance of the CSV species to human, we obtained the value in million 289 years since divergence from the TimeTree database (73). Given a human transcript $T$ and its 290 corresponding human gene $G$, we queried Ensembl BioMart for $G$ 's orthologs in the 20 species, $\mathbf{G}_{\text {orthologs }}$ $291=\left[G_{\text {yeast }}, G_{\text {worm }}, G_{\text {fruittly }}, \ldots, G_{\text {chimpanzee }}\right.$. We downloaded all coding DNA sequences (CDS) for these 292 orthologs from Ensembl (release-94) (74). For each gene in $\mathrm{G}_{\text {orthologs, }}$, we identified its longest transcript 293 per organism, $T_{\text {orthologs }}=\left[T_{\text {yeast }}, T_{\text {worm }}, T_{\text {fruittly }}, \ldots, T_{\text {chimpanzee }}\right]$. We then used PRANK (75) to generate a 294 multiple sequence alignment (MSA) for each T. PRANK aligns CDSs by first translating them into protein 295 sequences so that gaps tend to be placed between codons, instead of within codons. For each codon in 296 each human transcript, we could identify if other organisms carried the same codon or another, even if 297 the amino acid remained the same. If the codon was different, the corresponding human sSNV was 298 termed a CSV.

299 Evaluation of synVep predictions according to constraint on coding regions. Constrained regions 300 (76), referenced by genomic coordinates, were downloaded from https://s3.us-east301 2.amazonaws.com/ccrs/ccr.html. The constraint of human coding region is measured by percentile (of 302 residuals from a linear regression for distance-to-mutation prediction as computed in (76)), where a high 303 percentile indicates a more constrained region. We annotated the sparsity of sSNVs, i.e. the fraction of 304 observed sSNVs among all possible sSNVs in a region of a certain constraint level, and the median 305 synVep prediction of variants in these regions.

306 Analysis of sSNVs identified in Qatari Genome. We downloaded all VCF files containing variants 307 identified from the Qatari Genome project (QTRG) (77) from NCBI Sequence Read Archive (78) 308 (https://trace.ncbi.nlm.nih.gov/Traces/sra/?study=SRP061943). We then parsed these VCF files, 309 extracted the variants, and mapped the sSNVs to our observed, singleton, not-seen, and unobservable 310 sets. 


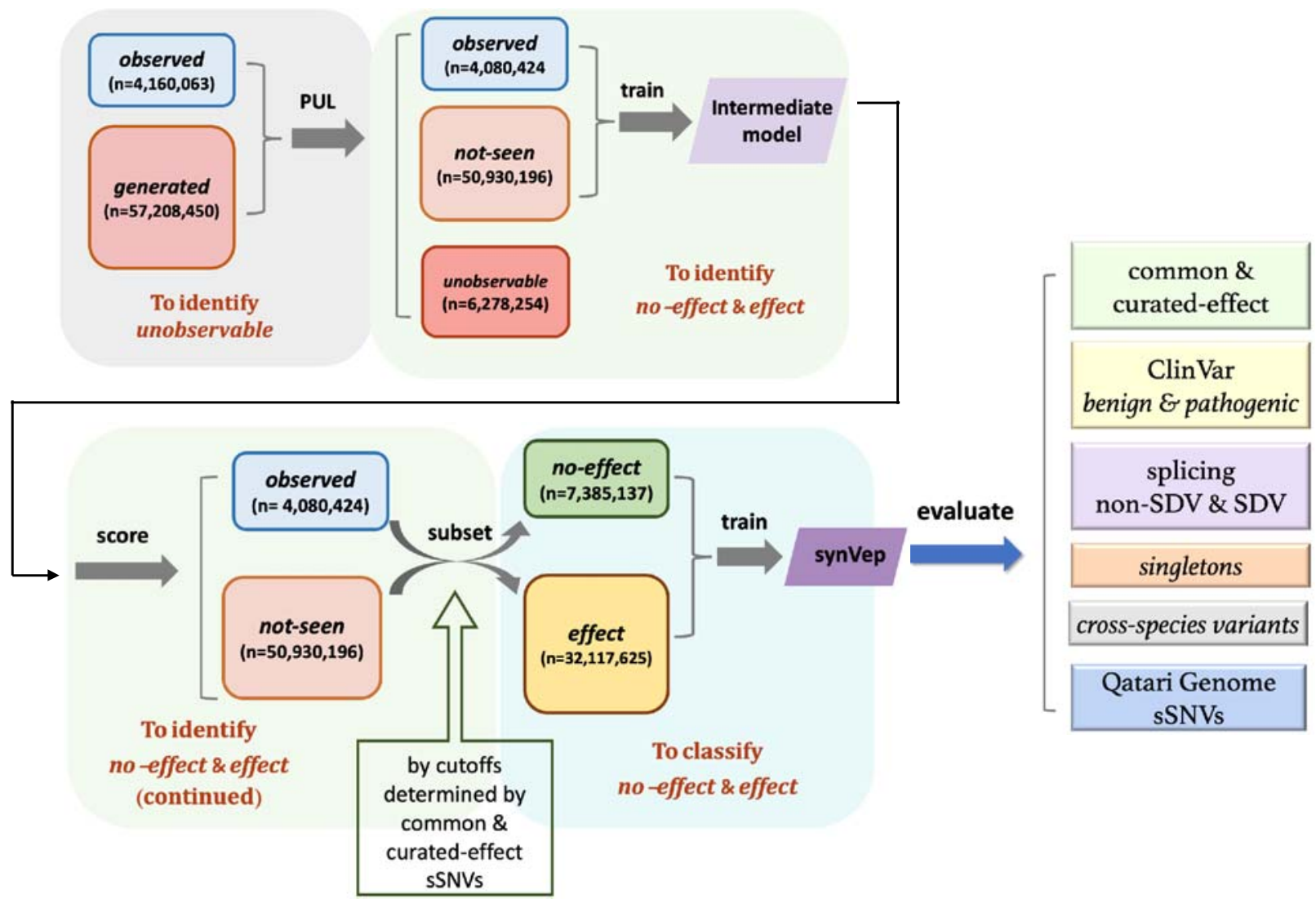

Figure 1. Pipeline of predictor construction. Starting with 4,160,063 observed and 57,208,450 generated sSNVs, 63 epochs of positive unlabeled learning (PUL) was conducted to separate the generated set into not-seen (observable) and unobservable set (Supplementary text). An intermediate model was trained using the observed and not-seen sets (observed set was up-sampled to equal amount of not-seen variants). The intermediate model's predictions for common and pathogenic sSNVs were used as guideline to set cutoffs assigning no-effect and effect set. The final predictor was trained using the no-effect and effect sets (no-effect set was up-sampled to equal amount of effect sets). After the final synVep model was trained, it was evaluated on independent datasets as shown. Here, singletons are sSNVs found in only one individual in gnomAD; observed are any other sSNVs found in gnomAD; generated are all possible sSNVs, except singletons or observed; unobservable are sSNVs PUL-labeled to be unlike function or quantity of a gene product. 


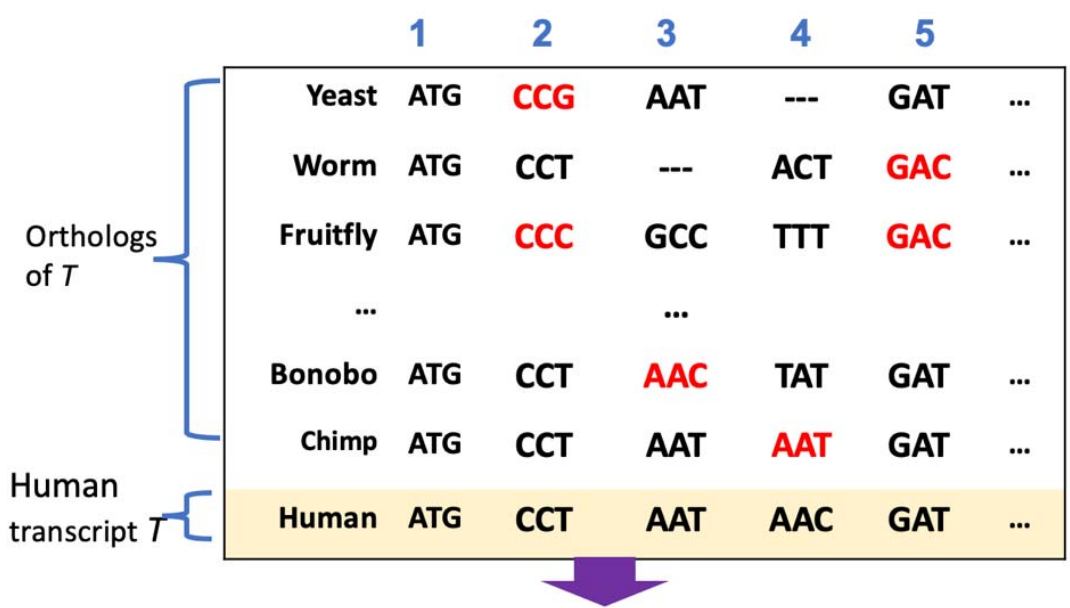

\begin{tabular}{|ccccccccc|}
$\begin{array}{r}\text { Human } \\
\text { transcript }\end{array}$ & $\begin{array}{c}\text { Codon } \\
\text { position }\end{array}$ & Variant & Yeast & Worm & Fruitfly & $\ldots$ & Bonobo & Chimp \\
$T$ & 2 & CCT>CCG & 1 & 0 & 0 & $\ldots$ & 0 & 0 \\
$T$ & 2 & CCT>CCC & 0 & 0 & 1 & $\ldots$ & 0 & 0 \\
$T$ & 3 & AAT>AAC & 0 & 0 & 0 & $\ldots$ & 1 & 0 \\
$T$ & 4 & AAC>AAT & 0 & 0 & 0 & $\ldots$ & 0 & 1 \\
$T$ & 5 & GAT>GAC & $\mathbf{0}$ & $\mathbf{1}$ & $\mathbf{1}$ & $\ldots$ & 0 & 0 \\
\hline
\end{tabular}

Figure 2. Extraction of cross-species sequence variants (CSV). For each human protein coding transcript $T$, codon-oriented multiple sequence alignment was performed with 20 species' longest coding sequencing of the same ortholog as $T$. The CSV are represented as "codon>codon" format for specific transcript positions and may coincide with human sSNVs.

\section{RESULTS AND DISCUSSION}

333 Generated sSNVs may be observable in the future. In the absence of a gold-standard experimentally 334 validated data set describing sSNV functional effects, we sought an alternative for the development of our method. We had previously proposed to use sSNVs that have been observed in major sequencing projects vs. all other possible human genome sSNVs (the generated set) for method evaluation (17). We collected 72,400 human transcripts with 4,160,063 ( $n=1,520,334$ genomic coordinate-based) observed sSNVs and 3,438,470 ( $n=1,233,878$ genomic coordinate-based) singletons (observed in only one individual) from the exome sequencing data of the Genome Aggregation Database (gnomAD exome) (39). We then created a generated set of 57,208,450 ( $n=21,314,668$ genomic coordinate-based) all possible sSNVs in these transcripts that were not found in gnomAD data. Note that only $\sim 12 \%$ of all SSNVs in our set were ever reported by gnomAD. We annotated these sSNVs with 35 transcript- and variant-specific features, including codon bias, codon autocorrelation, transcript stability, expression level, distance to regulatory sites, predicted protein secondary structures, etc. (Methods; Supplementary Table S2).

While the observed sSNVs are not necessarily functionally neutral, they are at least compatible with life. or may not become observed with more sequencing, and the unobservable ones, which cannot be 
observed given the contemporary variant-discovery capability. Note that the unobservable character of sSNVs may be due to a broad range of technical and biological reasons such as sequencing $(79,80)$, molecular functional constraints (81), and analytical biases or extreme deleteriousness resulting in early embryonic incompatibility with life $(82,83)$. We also note that in our modelling, the unobservable set may simply be poorly described by our selection of variant features.

354 We used observed sSNVs as positives in positive-unlabeled learning (PUL) (32-34) to differentiate the not-seen sSNVs (similar to observed) from unobservable ones in the generated (unlabeled) set (Figure

356 1). At convergence (epoch 63, Methods; Supplementary Figure 1), PUL partitioned all generated sSNVs

357 into unobservable ( $n=6,278,254$ transcript-based and 2,764,229 genomic coordinate-based; $11 \%$ ) and 358 not-seen ( $n=50,930,196$ transcript-based and 19,730,623 genomic coordinate-based; 89\%). Additionally,

$3598 \%(n=266,192)$ of singletons were deemed unobservable by the PUL model, as were $2 \%$ of the 360 observed sSNVs $(n=79,639)$. The latter result highlights the possible insufficiency of our variant 361 descriptors for capturing the complete observable variant diversity, while the former may also indicate 362 sequencing errors. The difference in percentages of variants misidentified by the model $(11 \%$ of 363 generated vs $2 \%$ of observed), however, suggests that deleteriousness of variants also plays a role in 364 defining unobservable variants.

Observed and not-seen variant sets contain both no-effect and effect sSNVs. We trained the intermediate model (Figure 1) to recognize observed vs. not-seen sSNVs. The model accurately (F-score=0.71; Eqn. 3) recognized the two classes in a previously unseen variant test set (Supplementary Figure 2). It also predicted $9 \%(9,282,542)$ of the not-seen SSNVs to be observed (scoring $<0.5)$, implying that these may be sequenced in the future.

370 Although large effect sSNVs may be enriched in the not-seen group, the intermediate model cannot be 371 directly used to evaluate effect, because it is only meant to predict whether an SSNV has been observed 372 or not. To build a model for effect evaluation, we leveraged the intermediate model's predictions on 373 common variants excluded from training and the experimentally validated effect sSNVs (curated-effect; 374 Methods; 170 transcript-based sSNVs). While these curated-effect sSNVs are, in fact, observed, their 375 prediction scores were higher than those of the excluded common set (Supplementary Figure 2, Mann376 Whitney $U$ test $p$-value<2.2e-16). This observation is likely due to the fact that the not-seen set is 377 enriched, while the common variant set is depleted, in large effect sSNVs. We assume this for common 378 variants because large-effect deleterious variants would not become common and large-effect 379 advantageous variants would tend to become wild-type.

380 We excluded $10 \%(7,957)$ of the common sSNVs from training of the intermediate model for selecting the 381 cutoff of effect/no-effect variants as next described. For training of the final model, we selected as no382 effect those sSNVs (both observed and not-seen) scoring below the intermediate model prediction 383 median (0.38) of the excluded common sSNVs; variants scoring above the intermediate model median of 384 the curated-effect sSNVs (score $=0.63$ ) were labeled effect (Supplementary Figure 2). We thus obtained 

and 31,712,455 not-seen) effect SSNVs. We trained the final model (synVep, Figure 1) to differentiate the no-effect and effect SSNVs (in balanced class training), using a 9: 0.5: 0.5 split of data for training, validation, and testing purposes (Methods). synVep was accurate ( $\mathrm{F}$-score $=0.90$; binary score cutoff $=0.5$ ) in evaluating the hold-out test set $(369,257$ no-effect and 369,257 effect). Note that synVep prediction scores did not correlate with allele frequency (Pearson correlation=0.02).

391 Feature importance in discriminating effect. We collected 35 features (Supplementary Table S2; Methods) 392 highlighting the different ways how sSNVs can impact gene function (17). We examined the correlation of 393 feature scores across all sSNVs (Supplementary Figure 3; Methods) and computed feature importance 394 for the final model (Supplementary Figure 4; Methods). Feature scores correlated within the same 395 feature category for some categories (e.g. codon context, codon bias, and expression profile), but not 396 across different categories. The most important feature for our model was codon_mutation (i.e. the wild 397 type/mutant codon pair), which is consistent with our earlier observation that some codons are 398 preferentially mutated in observed sSNVs (17) and with the Karczewski et al (39) observation that CpG399 transitions in the population are closer to saturation than other mutation types. Another codon context 400 feature - next_codon, which is highly correlated with the last_codon and codon_mutation features - was 401 the third most important feature. This reflects the biological importance of codon pairs in modulating 402 translational efficiency (84-86). Codon bias measures (and their changes due to mutation) were also of 403 high importance (starting at second highest rank), in line with the abundant evidence of the relationship 404 between codon composition and a variety of biologically-relevant factors, including gene expression $405(49,87,88)$, translational efficiency $(14,89,90)$, and mRNA stability (91-94). Since codon selection 406 modulates translational speed and thus cotranslational folding (95), sSNVs can also affect protein 407 structure (96) without altering protein sequence. We incorporated protein annotations (predicted 408 secondary structure, solvent accessibility, and disorder) as features; curiously, solvent accessibility 409 ranked $8^{\text {th }}$ in importance for the synVep model. Surprisingly, most other features had low importance; 410 including features related to mRNA structure and stability, which are known to be directly influenced by 411 sSNVs (8). This is perhaps due to the fact it is difficult to accurately predict RNA structure/stability for 412 sequences longer than 500 nucleotides (97), and over $74 \%$ of transcripts in our data are longer than that.

413 Note that we did not use conservation as a synVep feature as it is usually the overarching signal of effect 414 for most predictors (6) and we were hoping to capture additional, more subtle, signals orthogonal to those 415 already reported. However, we also evaluated synVep's potential performance loss due to this choice by 416 re-training the final model with an additional conservation feature (we used GERP++ scores for evaluation 417 purposes (69)). This model was not significantly better in discriminating no-effect/effect variants (0.9 vs $4180.9 \mathrm{~F}$-score with and without conservation, respectively, in evaluating the test set); we also note that the 419 difference in distribution of conservation scores across the effect and no-effect data sets was minimal 420 (Supplementary Figure 5). Driven by this somewhat unexpected lack of conservation difference between 421 the effect/no-effect sets, we further aimed to validate our set selection at the intermediate model level. We 
trained an intermediate model using conservation as one of the features. This model identified a new, conservation-included set of effect $(n=6,263,638)$ and no-effect $(n=32,010,049)$ sSNVs. This new partition overlapped significantly with the original one $(75 \%$ of the original sSNVs were present and had identical effect/no-effect labels in both data sets). Moreover, synVep (without the conservation feature) predicted both data sets equally well. Using a balanced test set from the original data, it achieved $89 \% / 90 \%$ noeffect precision/recall (Eqn. 1 and Eqn. 2) and 90\%/89\% effect precision/recall; it achieved a similar performance for a balanced subset of the conservation-included effect/no-effect set $(87 \% / 88 \%$ no-effect precision/recall and $87 \% / 88 \%$ effect precision/recall). Given these results, we chose to further continue to exclude conservation from synVep features. This choice makes synVep scores orthogonal to those of other predictors and allows for the further described cross-species variant analysis to be performed.
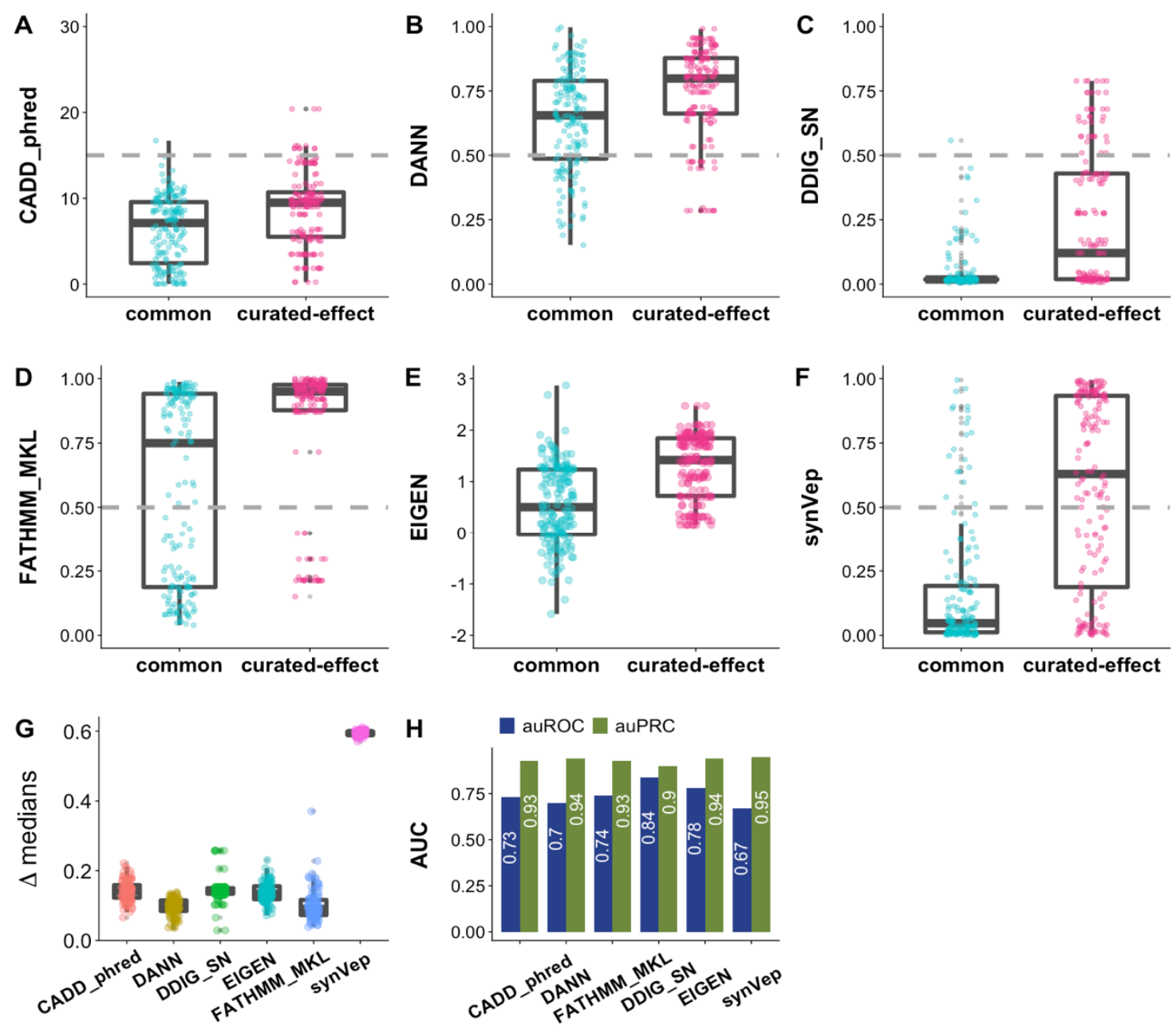

433

435

436

437

438

439
Figure 3. Predictor performance on common vs. curated-effect sSNVs. Panels A-F show the differential predictions on sets of curated-effect $(n=170)$ and common sSNVs (randomly selected $n=170$ ) for CADD (phred-like scaled scores), DANN, DDIG-SN, FATHMM-MKL, EIGEN, and synVep, respectively. Gray line indicates scoring cutoff suggested by tool authors. Neither the common set nor the curated-effect set were included in synVep training. In panel (G), this comparison was repeated 100 times to compute the medians (i.e., median $_{\text {curated-effect }}-$ median $\left._{\text {common }}\right)$. Permutation tests show that all predictors give significantly different scores between the two sets in 
every iteration, except for DANN where 11 of 100 comparisons were not significant (p-value $>0.05$ after Bonferroni correction). Panel $\mathrm{H}$ reports the performance (auROC and effect auPRC) of each predictor on the left-out common sSNVs (negative; $n=9,274$ ) and curated-effect sSNVs (positive; $n=170$ ). All other predictors' (except CADD_phred and DANN) auROC and auPRC are significantly different from synVep's (p-value $<0.05$; Methods). Note that the utility of auROC and auPRC is limited in single variant effect prediction; instead, a cutoff is needed.

Predictors identify sSNV effect. To evaluate the performance of synVep, we needed a gold standard set of designated effect and no effect variants. However, since there is no experimentally validated "neutral" sSNVs, we used the common sSNVs excluded in training as neutrals (no-effect), i.e. as described above we assumed that the majority of common sSNVs have little effect. On the level of molecular function, the effect of a variant is not directional (neither advantageous nor deleterious). On the level of individual fitness (evolutionary), however, variants could be advantageous, neutral, or deleterious. Note that evolutionarily effect variants must have a molecular effect, but not vice versa. The neutral theory of molecular evolution, as well as some experimental work, suggest that advantageous mutations are rare, and most effect mutations are deleterious $(98,99)$. Variants with large deleterious effects on evolutionary fitness would have been purified out and will not be seen in the population. Neutral or weak effect mutations could, depending on the population size, become common or even fixed due to genetic drift (98). Thus, in the absence of experimentally neutral variants, common variation may be considered a reference point for neutral or weak effect variants.

We used the set of curated-effect sSNVs ( $n=170$; excluded from synVep training) as the effect group and the subset of common sSNVs left-out from synVep training $(n=9,274)$ as the no-effect group to compare the predictor performances (Figure 3). Of the considered methods, synVep had the highest auPRC but the lowest auROC (Figure $3 \mathrm{H}$ ), suggesting that other predictors' decision cutoffs may not be optimized for SSNV effect classification. Note that the utility of auROC and auPRC is, however, questionable in application to individual variants; here, a default cutoff is required to prioritize a variant. We note that most predictors failed to differentiate the two groups of variants at the default binary cutoff, placing both sets of variants below (CADD, DDIG-SN) or above the default threshold (DANN, FATHMM-MKL). Although possibly helped by the intermediate model choices, the majority of synVep's predictions for the two groups fall on two sides of the default cutoff.

We further designed an additional testing scheme to evaluate predictor performance. Since the test set of no effect common sSNVs $(n=9,274)$ greatly outnumbered the curated-effect set $(n=170)$, we randomly sampled 170 sSNVs from the common set 100 times; each time, we computed the difference between the effect-median and no-effect-median scores (Methods). synVep outperformed other predictors (Figure 3F; CADD and EIGEN scores normalized to 0-1 scale), although all predictors identified most curatedeffect SSNVs as having more effect than common SSNVs. Note that the "general-purpose" predictor (CADD, DANN, and EIGEN) cutoffs are not optimized to specifically evaluate synonymous variants and may thus be expecting a larger signal of effect. 
477 We also examined the correlation of the predicted scores (Figure 4A) and the Fraction of Consensus 478 Binary Prediction (17) (FCBP; Figure 4B) on all 4,160,063 observed sSNVs for all predictors (synVep, 479 CADD, DANN, FATHMM-MKL, DDIG-SN, and EIGEN). synVep's scores were poorly correlated with other 480 predictor scores (Pearson correlation ranging from - 0.1 [DANN] to 0.23 [FATHMM-MKL]), while binary 481 classification was more similar (FCBP ranging from 0.37 [DANN] to 0.69 [CADD and DDIG-SN]).

A

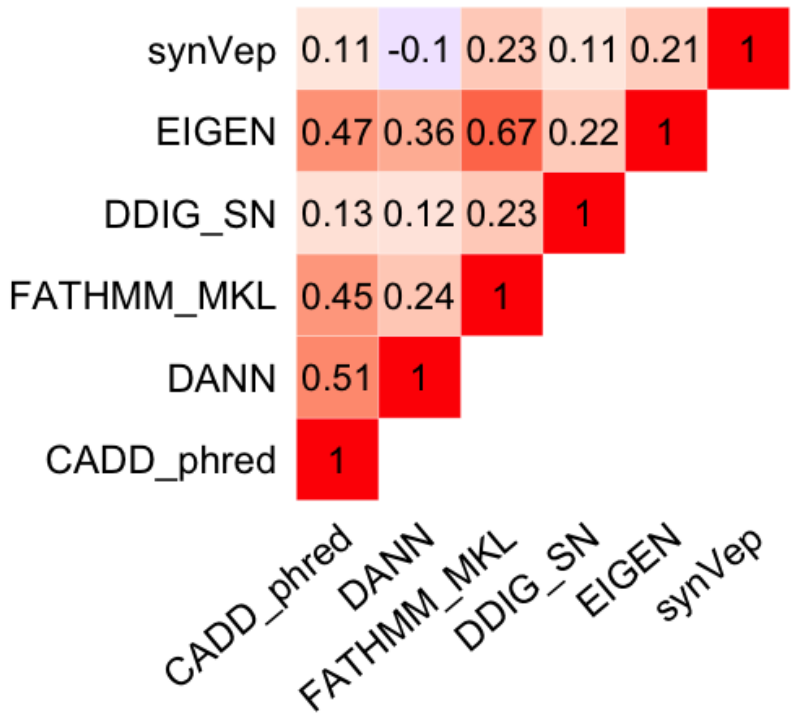

B

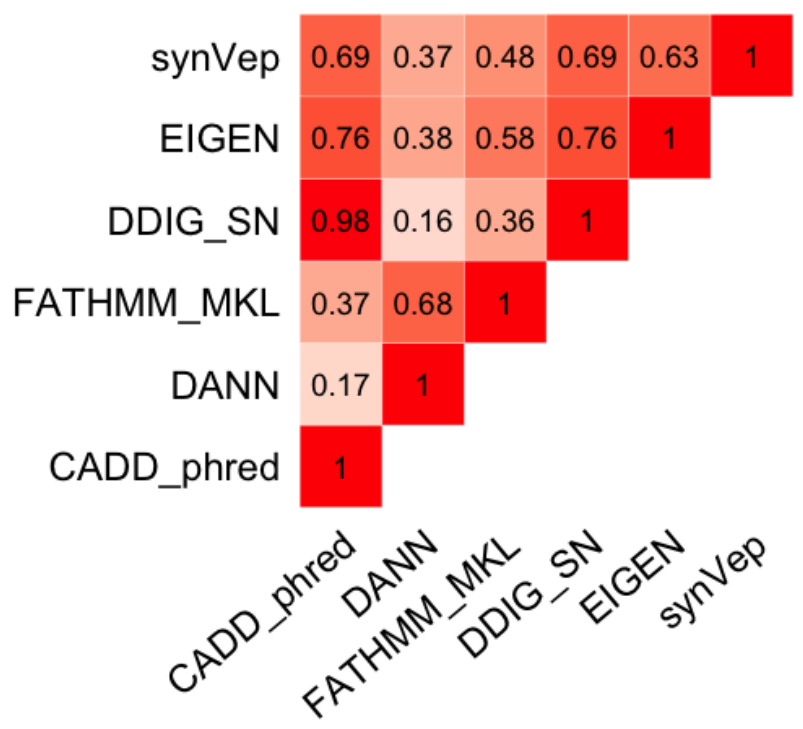

Figure 4. Comparing predictions made by synVep and other tools. Panel (A) Spearman correlation and (B) Fraction of Consensus Binary Prediction (FCBP) highlight similarity and lack thereof among the predictors for all observed sSNVs $(\mathrm{n}=4,160,063)$.

Singletons are more likely than observed to have an effect. We re-predicted scores of all variants in our data (excluding unobservable) with the final synVep model. As expected, of observed sSNVs, only 31.3\% were effect (median score 0.15 ), while $72.0 \%$ of not-seen sSNVs were effect (median score 0.88 ); singletons were scored/distributed bimodally (Supplementary Figure 6) into the two classes (48.2\% effect; median score 0.47 ). Note that synVep predictions did not appear to be driven by site mutability (putative mutation rate). synVep scores of not-seen SSNVs that share a genomic position with none, 1, or 2 observed variants do not significantly differ from each other (median synVep scores 0.86, 0.94, and 0.88 respectively; Supplementary Figure 7 ).

Singletons were not included in our training because it is difficult to estimate how many of them are artifacts due to the $0.1-0.6 \%$ error rates of next-generation sequencing (100). If the singletons are not artifacts, then they are likely to be individual or ultrarare variants. These are more likely to be effect than higher frequency variants $(101,102)$. An excess burden of ultrarare variants (although not necessarily 
synonymous) is also often seen in diseases, such as schizophrenia (103-105), Parkinson disease (106), and bipolar disorder (107). In line with these expectations, we found that singletons were, on average, scored higher than observed sSNVs (Supplementary Figure 6), suggesting that singletons are more likely to have an effect than the observed.

$503 \quad$ Variant effect predictors differentiate benign and pathogenic variants. Among the predictors considered in 504 this work, only two (FATHMM-MKL and DDIG-SN) are explicitly aimed to assess variant pathogenicity. To 505 investigate whether predictors for variant functional effect (i.e. not pathogenicity) can identify pathogenic 506 sSNVs, we obtained from ClinVar 17 pathogenic (genomic coordinate-based, 68 transcript-based) sSNVs 507 and 51 benign sSNVs (genomic coordinate-based, 254 transcript-based) variants reviewed by an expert 508 panel. Of these 68 variants, one benign and one pathogenic (genomic coordinate-based, 13 transcript509 based) were deemed unobservable by our model and were removed from consideration. Note that for 510 fairness of evaluation, these ClinVar sSNVs were also excluded from training of synVep. All variant-effect 511 predictors, including synVep, assigned higher scores to Pathogenic than Benign variants (Figure 5A-C 512 and E). However, at the default/recommended cutoff, only synVep placed the majority of Benign vs. 513 Pathogenic variants on opposite sides of the cutoff (Pathogenic recall=0.58; Benign recall=0.66).

514 synVep also attained the highest effect auPRC (Figure 5F), suggesting that it can identify disease515 causing SSNVs well even though it was not explicitly trained to do so. However, synVep attained the 516 lowest auROC, which may be due to the fact that benign ClinVar variants are actually functionally 517 significant (effect) and are thus predicted by synVep as such but classified as wrong by ClinVar 518 annotation (FP). In our definition, a variant of some effect is not necessarily pathogenic, but pathogenic 519 variants are expected to have effect. Thus, experimentally validated pathogenic variants predicted to be 520 no-effect by synVep are likely errors, but benign variants predicted be effect are quite possibly correctly 521 identified as having functional impact, which does not necessarily correspond to disease.

522 Note that because synVep's predictions are transcript-based, they can differ for the same variant 523 across multiple transcripts. Aggregating these predictions to score a variant is not trivial: one can 524 use the mean, maximum, or median scores. A more sophisticated approach would be to weigh the 525 scores from different transcripts by their expression level in multiple tissues. Specifically, if the 526 question is about a disease and if the disease is primarily associated with one tissue, only the 527 transcript most expressed in that tissue cab ne considered. However, given the complicated 528 regulation and genetic interactions, this idea needs further validation. An evaluation of predictions for 529 the same variant, however, highlights an interesting observation: only $5.1 \%$ of not-seen, $5.5 \%$ of 530 observed, and $7.4 \%$ of singletons had at least one transcript, whose effect prediction differed from 531 others. 

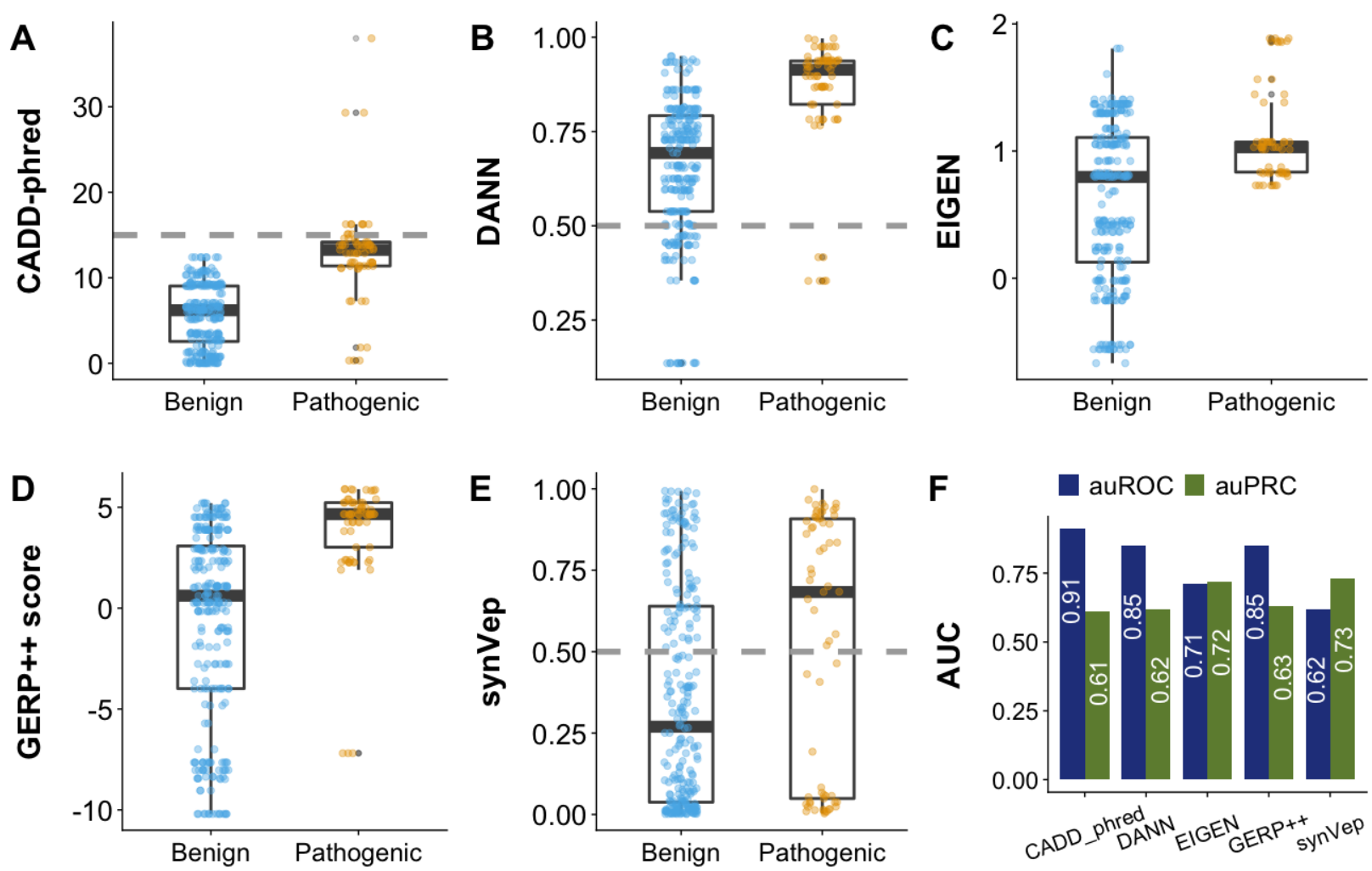

Figure 5. Evaluating variant effect predictors using ClinVar data. Benign (negative; n=254) are variants labeled "Benign" and "Benign/Likely benign" in ClinVar, with "Review by expert panel" as review status. Pathogenic (positive; $n=68$ ) are those labeled "Pathogenic" and "Pathogenic/Likely Pathogenic" in ClinVar, with "Review by expert panel" as review status or with "research" method and at least one publication experimentally validating the effect. Panel A-E show the predictions from for CADD (phred-like scaled scores), DANN, EIGEN, GERP++ score, and synVep, respectively. Grey dashed lines show the method author-recommended cutoffs, where available. Differences between scores of Benign and Pathogenic variants in panels A-E are all statistically significant (onesided permutation test $\mathrm{p}$-value $=0$ ). Panel F reports auROC and auPRC for each predictor; all predictor auROCs and auPRCs are significantly different from that of synVep (p-value<0.05; Methods). Note that the utility of auROC and auPRC is limited in single variant effect prediction; instead, a cutoff is needed.

All variant-effect predictors, including synVep, assigned higher scores to pathogenic than benign variants (Figure 5A-C and E, all statistically significant, one-sided permutation test $p$-value $=0$ ). Notably, conservation (GERP++ (69)) carried sufficient signal to recognize pathogenic variants as well, suggesting that these are often found in conserved positions, which may not be the case for variants of less severe effect. Note that all other predictors (CADD, DANN, and EIGEN) incorporate GERP++ as a feature, but their auROC and auPRC are not substantially higher (or even lower) than those of GERP++. Highly conserved genomic positions often have experienced extensive purifying selection (108). Therefore, conservation is understandably a commonly used feature for disease variant prioritization (109). However, in the scenario of disease variant prioritization synVep, offers discriminative power independent of conservation, so it may be used in combination with a conservation score or other predictors. 
555 One major challenge in disease variant prioritization is that for complex diseases, causality can rarely be 556 explained by a single variant (110). The utility of variant pathogenicity score is thus questionable: does a 557 high score suggest a high likelihood of an individual developing a disease or a high likelihood of this 558 variant contributing to a disease? Also, would an individual with many predicted-pathogenic variants carry 559 many diseases or be very certain to carry at least one disease? A potential way to solve this puzzle is to 560 establish the variant-disease relationship using the collective effect from the whole variome instead of a 561 single or a few variants. A modification of polygenic risk scoring methods $(111,112)$ to only account for 562 effect variants may represent one approach, although it would be limited by the location of most GWAS 563 SNPs in non-coding regions. Another approach is to unite only the coding variant effects by aggregating 564 all variants per gene to predict disease predisposition (e.g., $(113,114)$ ) synVep predictions (as well as 565 those of other predictors) may be plugged in these pipelines to explore the contribution of sSNVs to 566 complex diseases.

567 synVep highlights correlation between conservation and effect. We annotated all sSNVs as CSVs (cross568 species variation) or not (Figure 2; Methods). CSVs are codon differences between the human reference 569 sequence and another species' ortholog. For example, if the proline-coding codon in a human transcript $T$ 570 is CCC, while the aligned proline codon on Ts chimp ortholog is CCT, then the human SSNV CCC->CCT 571 is considered a chimp-CSV. We thus annotated 15,618,155 unique (only exists in one species) and $57235,102,565$ non-unique (overlapping across species) CSVs (Supplementary Figure 8). Since less than $57310 \%(7,026$ of 72,400$)$ of the human transcripts can be mapped to orthologs in all 20 species, we 574 analyzed separately the CSVs in (1) all transcripts $(n=32,264,860)$ and (2) only the transcripts that have 575 orthologs in all 20 species $(n=3,321,574)$ and that are likely ancient (ancient genes) $(115)$. 
A

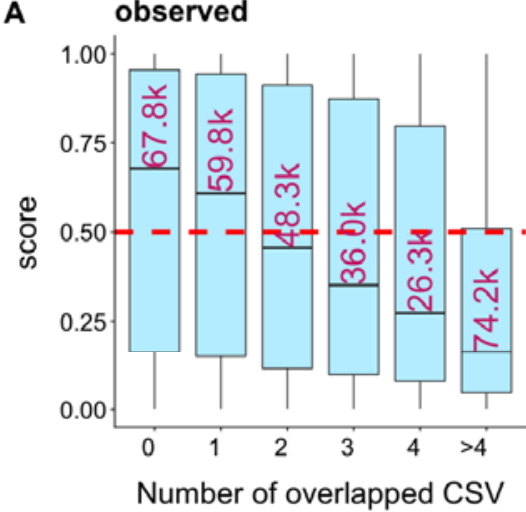

D

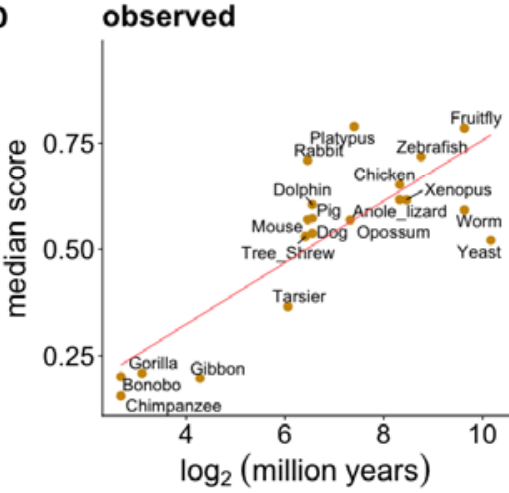

B

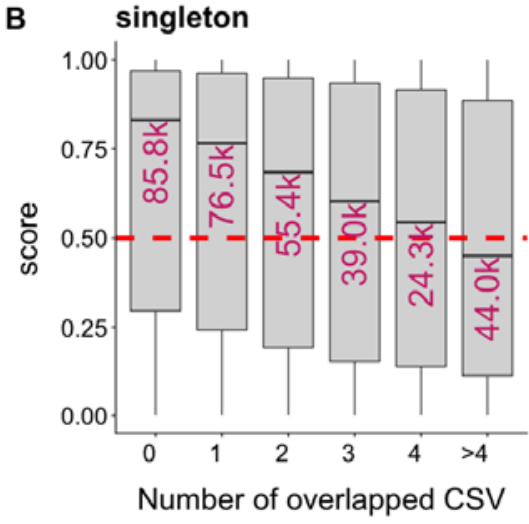

E

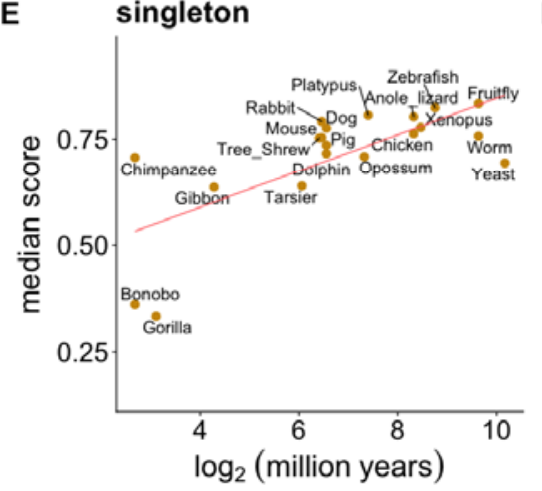

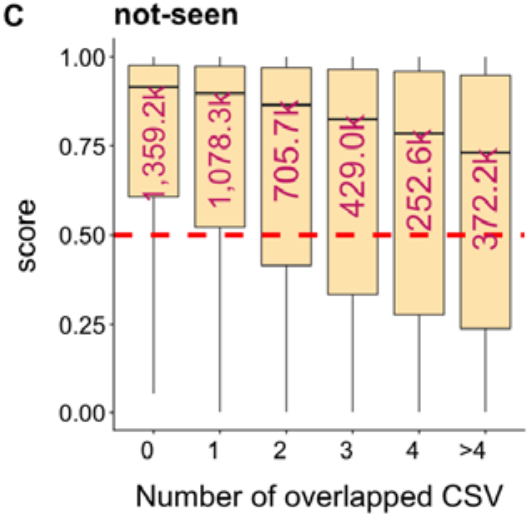

$\mathbf{F}$

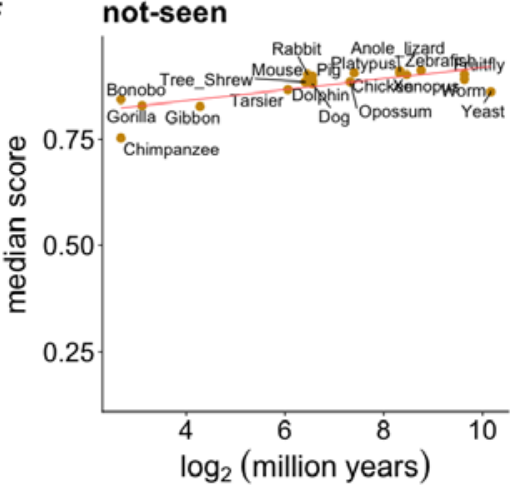

578

579

580

581

582

583

584

Figure 6. Variant effect prediction from the perspective of cross-species variation (CSV). Panels A-C show synVep-predicted scores for variants grouped by the number of species carrying the mutant nucleotide; separately for observed, singletons, and not-seen sets. The red dashed line is synVep's default cutoff for effect and no-effect. The number in each box indicates the number of variants of that group (in thousands). Panels D-F show the median score (y-axis) across species at $\log _{2}$ million years since divergence from common ancestor with human (x-axis) and linear regression trendline (red line) between the two. The Spearman correlations between median synVep score and $\log 2$ (million years) for panel D-F are $0.68,0.64$, and 0.66 , respectively.

The distribution of synVep prediction scores for CSVs in the ancient genes and for those in all transcripts were similar ( mean=0.05, Mann-Whitney $U$ test $p$-value<2.2e-16), suggesting that synVep's evaluation of variants does not discriminate by gene age. For all transcripts, observed sSNVs had more CSVs $(67 \%$, $n=2,823,142)$ than did the not-seen variants $(53 \%, n=26,976,016$; Supplementary Figure 9$)$. CSVs overall were predicted less likely to be effect [reviewer3] than non-CSV for both ancient and all transcripts (Figure 6 A-C; Supplementary Figure $10 \mathrm{~A}-\mathrm{C}$ ). While this is in line with the scoring trends of the observed and not-seen variants overall, it also mirrors earlier findings of few CSV nsSNVs corresponding to a known human disease (116-119). synVep scores also trended lower for CSVs whose substituting nucleotide was found in more species, for both ancient (Figure $6 \mathrm{~A}-\mathrm{C}$ ) and all transcripts (Supplementary Figure $10 \mathrm{~A}-\mathrm{C}$ ). Since the number of CSV species is somewhat indicative of codon conservation, this trend suggests that, although synVep was trained without using conservation features, its predictions still identify conserved codons that are often functionally relevant (120). 
597 To further elucidate the effect of sequence conservation across species, we calculated codon mutation 598 fraction (CMF, Supplementary Eqn. 9) to describe how common a human's alternative codon is, 599 compared to the reference codon, among the 20 species included for CSV analysis. For example, if in a 600 multiple sequence alignment of the 20 species orthologs, the human CCC codon is aligned to 10 CCC, 5 $601 \mathrm{CCT}$, and 5 other codons, then the CMF of the corresponding synonymous variant, CCC>CCT, is $6025 / 15=0.33$. We observed that predicted scores generally decrease with higher CMF (Supplementary 603 Figure $11 \mathrm{~A}-\mathrm{C}$ ), indicating that SSNVs with alternative codons commonly present as reference codons 604 among other species have less effect.

605 We additionally investigated the relationship between the evolutionary distance of CSV species from 606 human and the effects of the corresponding sSNVs. Since one sSNV can correspond to multiple species 607 CSVs, we only considered CSVs that are uniquely found in one species for this evaluation. The medians 608 of synVep scores of these species-exclusive CSVs in both ancient genes (Figure 6 D-F) and all 609 transcripts (Supplementary Figure $10 \mathrm{D}-\mathrm{F}$ ) correlated with the evolutionary distance of the corresponding 610 species to human. However, for ancient genes, the median scores of observed variants unique to further 611 related (i.e. beyond Tarsier) species were in the effect range (synVep>0.5). Arguably, this means that 612 human sSNVs that introduce nucleotides likely present in recent ancestors tend to be no-effect, while 613 similarity to further removed relatives carries no such benefit (Figure 6 D). These findings agree with our 614 recent work on nsSNV CSV analysis (35). We note that species relationship had much less impact on 615 binary effect classification for singleton variants and none for not-seen variants (Figure 6E-F). The same 616 observations could not be made for the all transcript set of variants, where observed and singleton CSVs

617 were predicted to be no-effect for a large portion of species (Supplementary Figure $10 \mathrm{D}-\mathrm{F}$ ). This 618 observation suggests that ancient genes are functionally crucial and have been sufficiently optimized over 619 time to only permit minor levels of variation without impact on functionality.

620 synVep differentiates splice-disrupting variants. Cheung et al. (70) measured the splice-disrupting effects 621 of genomic variants (3,297 transcript-specific sSNVs) and defined a group of large-effect splice-disrupting 622 variants (140 SDV sSNVs). As expected, synVep scores of SDVs were on average higher than those of 623 non-SDVs (Figure 7 A-C). Curiously, 140 SDVs comprised only six observed (4.6\%) and 18 singletons 624 (13.7\%) variants; nine were deemed unobservable (6.4\%) and 107 were not-seen (76.4\%). The fact that 625 most of SDVs are not-seen reinforces our assumption that not-seen sSNVs are enriched for large-effect 626 deleterious sSNVs that may have been purified. 

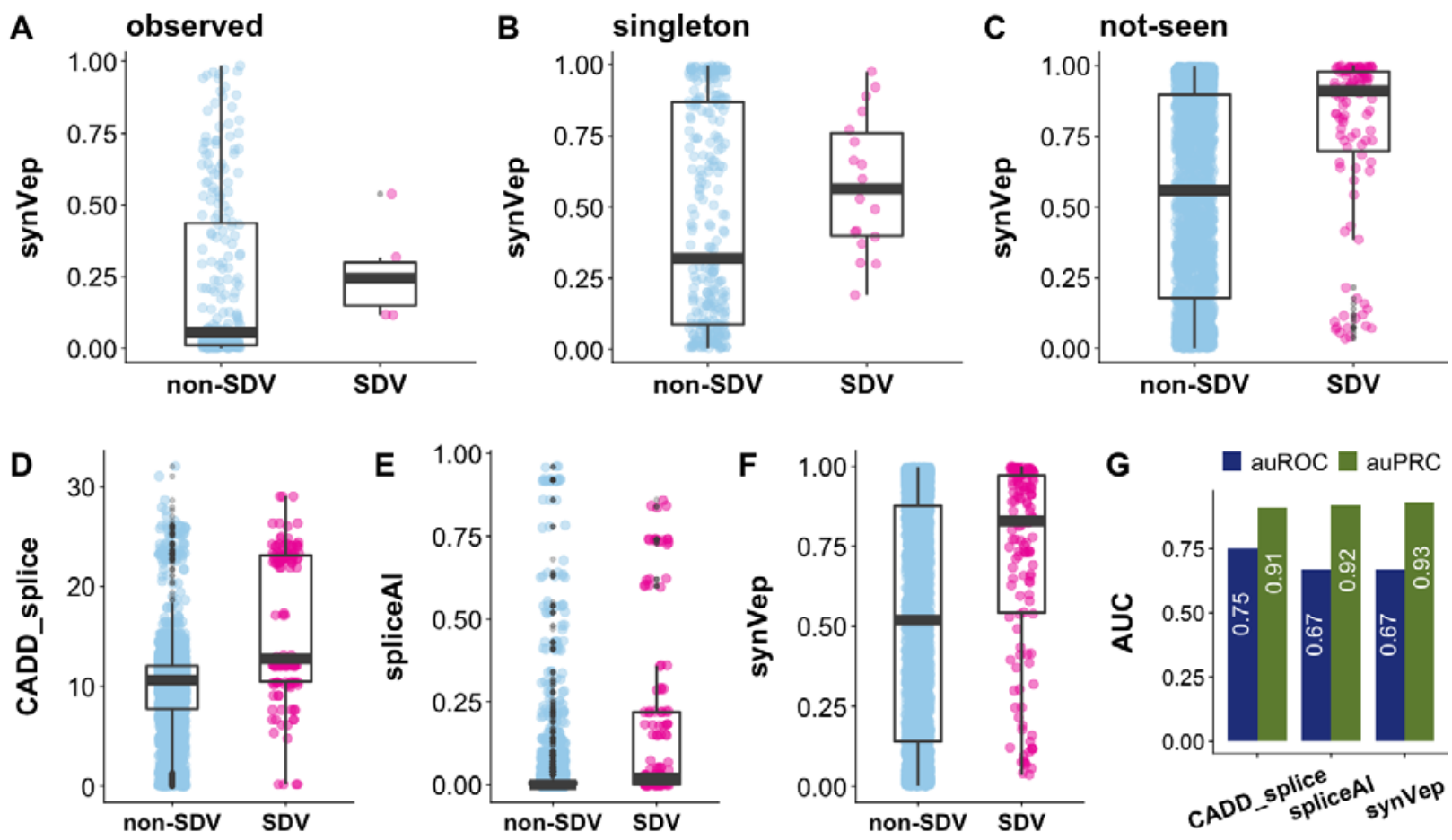

628

629

630

631

632

633

634

635

636

637

638

639

640

641

642

643

644

645

646

647

648

649

650

651

Figure 7. Evaluation of synVep, CADD-splice, and spliceAI using large-effect splice-disrupting variants (SDVs) and non-SDVs. SynVep predictions are higher scoring for a set of experimentally determined SDV (positive; $n=140$ ) than non-SDV (negative; $n=3,157)$ variants across observed $(\mathbf{A})$, singleton $(\mathbf{B})$, and not-seen $(\mathbf{C})$ data sets. Note that non-SDVs may still carry other functional effects. The observed SDVs mispredicted as no-effect highlight the limitations of our training data, although two of the five (40\%) observed SDVs are correctly annotated as effect. Panels D-F show the distribution of scores on complete non-SDV and SDV collections predicted by CADD-splice, spliceAI, and synVep, respectively. Panel G reports tha auROC and effect auPRC measures. CADDsplice auROC and auPRC are significantly different from synVep (p-value $<0.05$; Methods); while spliceAI's are not.

We evaluated two state-of-the-art predictors for splicing effect evaluation: CADD-splice (72) and spliceAI (71) on this set of experimentally determined variants (Figure 7D-G). spliceAl is a 32-layer deep learning model that predicts splicing donor/acceptor gain/loss probabilities. CADD-splice was developed based on CADD (23) with the addition of two splicing-specific predictor (MMsplice (121) and spliceAI) outputs as features. MMsplice and spliceAI were selected to be incorporated into CADD-splice because they performed best among several other splicing-specific predictors on the same non-SDV/SDV dataset (not limited to synonymous variants). CADD-splice has higher auROC (0.75) than both spliceAI (auROC=0.67) and synVep (auROC=0.67); meanwhile, the auRPC of all the three predictors are similar (Figure $7 \mathrm{G}$ ).

Splicing disruption is a well-known and well-studied mechanism of sSNV effect (122). In fact, most of the experimental validations of our curated-effect and ClinVar pathogenic variants refer to elucidating splicing effects (Supplementary Table S1 and S3). Moreover, many cancer driver mutations are found to be splice-disrupting synonymous variants (123). Aside from splicing, experimental validation of variant effect is rare, arguably due to technical challenges (124). Perhaps, since the experimental evidence for splicing disruption is more abundant than non-splicing effects', the former is considered a major factor in clinical 
652 consideration for sSNVs. For example, according to the guidelines from American College of Medical

653 Genetics and Genomics (125), an sSNV is clinically benign if it is not in a conserved position and is 654 predicted to be non-impacting to a splice site (e.g. via GeneSplicer (126), NNsplice (127)). Thus,

655 synVep's ability to identify effect and score sSNVs regardless of their splice effects or conservation

656 makes it an ideal tool for prioritization of all possible variants, regardless of their mechanism or

657 evolutionary evidence of effect.

658 sSNV effects reflect genomic constraints. Havrilla et al. developed the concept of "coding constrained 659 regions" (CCR) to describe the regional scarcity of protein-changing (missense or loss-of-function) 660 variants in the human genome (76). Here, a region with fewer of these variants observed in the human 661 population has a higher CCR percentile score. For our set of variants, the fraction of observed (number of 662 observed sSNVs divided by all possible SSNVs in this region) negatively correlated (Pearson $p=-0.61$ ) 663 with CCR percentile (Figure 8 A); i.e. higher constraint indicates fewer sSNVs. Furthermore, synVep 664 predictions positively correlated with CCR percentiles for observed ( $\rho=0.58$, Figure 8 B), i.e. lower CCR 665 percentile (less constrained regions) indicated lower (no-effect) synVep scores.
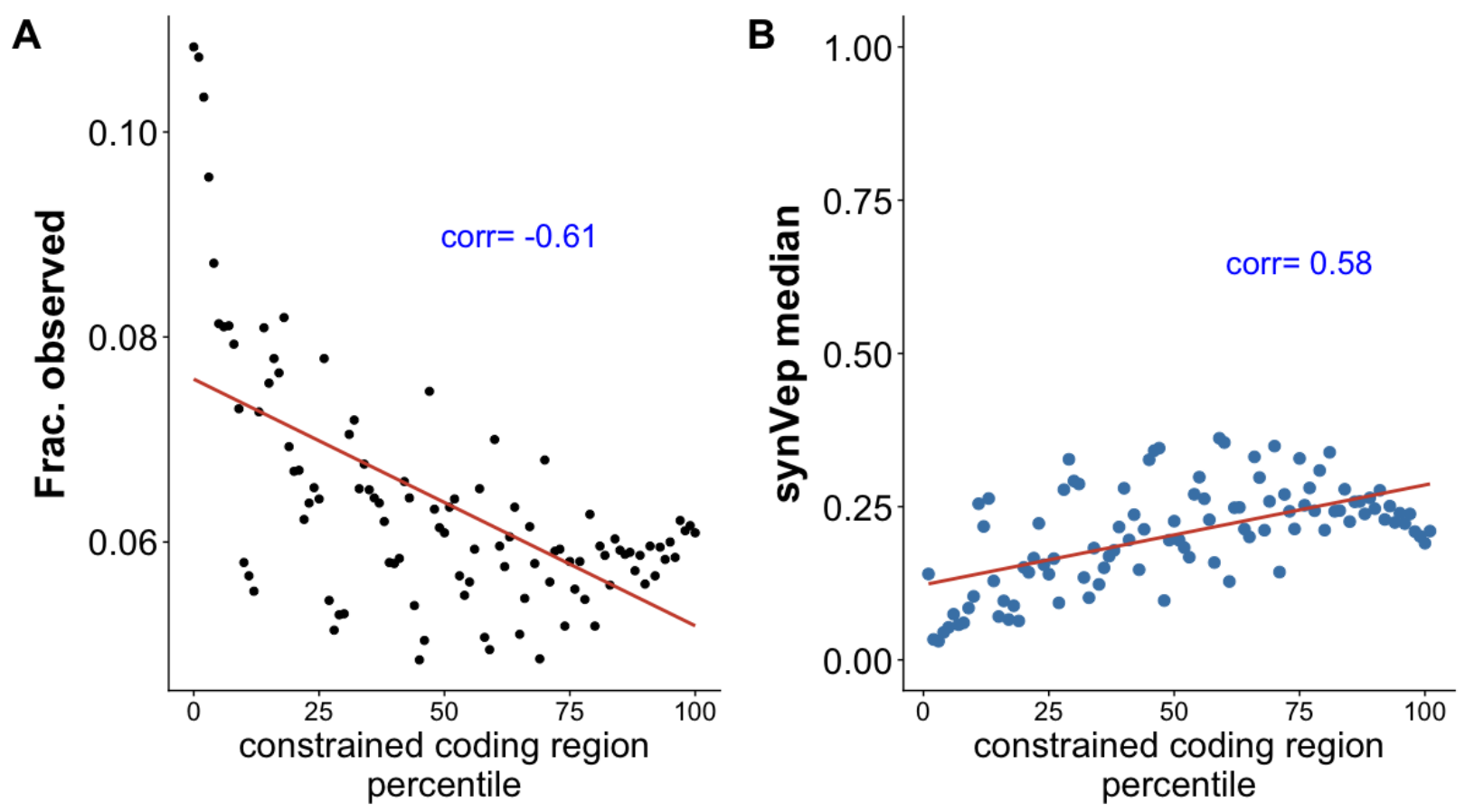

666

667

668

669

670

671

672 The negative correlation between the fraction of SSNVs and CCR indicates a positive correlation between

Figure 8. sSNV effect measured by region constraint. Coding constrained regions (CCR) describe the regional scarcity of nsSNVs; higher percentile regions represent have fewer observed nsSNVs. Observed sSNVs are relatively scarce in constrained regions $(\mathbf{A})$, while their median synVep scores are higher (B). Pearson correlation are indicated in blue.

\footnotetext{
673 synonymous mutation rate and missense or loss-of-function mutation rate. This observation is in line with
} 
674 earlier studies $(128,129)$, but raises a question of the utility of Ka/Ks ratio (non-synonymous divided by 675 synonymous mutation rate), which is widely used to measure the strength of evolutionary selection at 676 certain genomic sites (130). The application of the $\mathrm{Ka} / \mathrm{Ks}$ ratio is based on the assumption that 677 synonymous mutations are neutral and thus Ks can serve as a baseline for Ka. However, it has been 678 demonstrated that a high $\mathrm{Ka} / \mathrm{Ks}$ can also result from a low Ks due to strong negative selection at the synonymous sites $(10,11,131-133)$. Efforts have been made to improve the utility of $\mathrm{Ka} / \mathrm{Ks}$ by 680 incorporating codon preference (134-136), but the question remains: how often is the selection at 681 synonymous sites sufficiently underestimated so that $\mathrm{Ka} / \mathrm{Ks}$ is no longer accurate? Lawrie et al. found 682 that $22 \%$ of the fourfold synonymous sites (where the amino acid can be encoded by four codons) in the 683 fruitfly genome are under strong selection (137). Lu and Wu estimated that $90 \%$ synonymous mutations 684 in human and chimp are deleterious (138). Hellmann et al. estimated that $39 \%$ mutations at the human685 chimp-diverged non-CpG fourfold synonymous sites have been purified (139). Zhou et al. showed that $9 \%$ 686 of all yeast genes and $5 \%$ all worm genes undergo purifying selection on synonymous sites (136). In turn, 687 our results show that, excluding unobservable (9.6\%), $\sim 67 \%$ of all possible human sSNVs are effect 688 (synVep score>0.5), but we cannot estimate the strength of selection acting upon these. These findings 689 suggest that $\mathrm{Ka} / \mathrm{Ks}$ measures of genomic site constraints may be underpowered.

690 synVep sheds light on future variant discovery and interpretation. Whenever a human genome variant is 691 sequenced, it will automatically be reassigned a class in our collection. Thus, a newly sequenced variant 692 will first become a singleton and may, eventually, be a member of the observed group. An enrichment in 693 observed variants will likely come from large-scale sequencing. The ethnic diversity of gnomAD 694 represents the ethnic diversity in the United States, but not the global ethnicity diversity; although only $69516 \%$ of global population are of European descent (140), 53\% of the samples from gnomAD exomes 696 database are (141) are; i.e. there is a significant underrepresentation of sSNVs from other ethnicities. 697 When more diverse genomes are sequenced, will there be a significant addition to the observed set (i.e. 698 significant reduction of the not-seen set)?

699 To answer this question, we obtained all variants from the Qatar Genome (QTRG) project (77) and 700 mapped them to our set of SSNVs. QTRG comprises 1,376 individuals and may serve as a representative 701 pool of genomic variants in Middle East and north Africa (MENA) area (77); thus, this set is 702 complementary to gnomAD. We identified 526,616 transcript-based sSNVs ( $n=192,246$ genomic 703 coordinate-based) from QTRG sequencing. Importantly, only $0.6 \%$ of the Qatari sSNVs mapped to our 704 unobservable set - a fraction that is lower than the misprediction rate (5\%) that we allowed during PUL. 705 Moreover, two thirds of these variants were singletons in QTRG. This observation suggests that our 706 unobservable variants are indeed unlikely to be ever observed in future sequenced human populations. 707 The majority of QTRG sSNVs (81.9\%) mapped to our observed set; $4.6 \%$ and $12.8 \%$ were singleton and 708 not-seen, respectively (Figure 9A). Interestingly, 63.5\% and 64.6\% QTRG sSNVs mapping to our 709 singleton and not-seen sets, respectively, were singletons in the Qatari cohort. We also found that 710 gnomAD singletons that were present in QTRG, on average, scored higher than QTRG variants 

confirms that singletons are more likely to have an effect than observed variants.

713

A

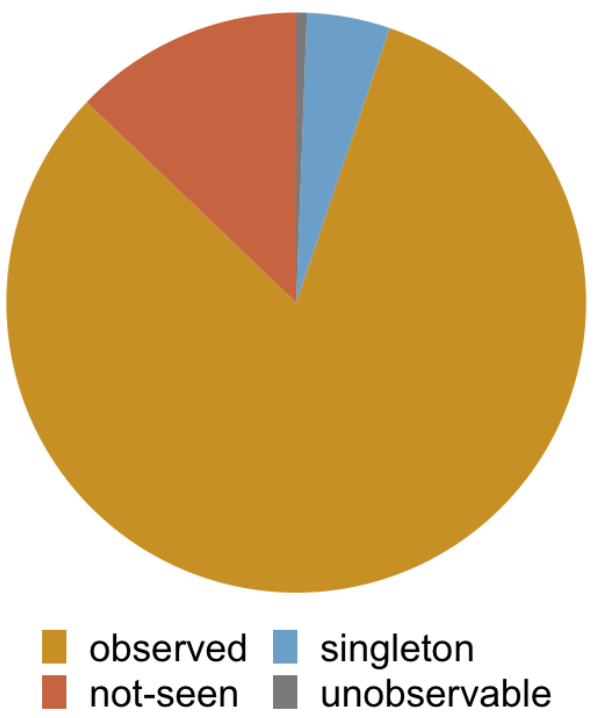

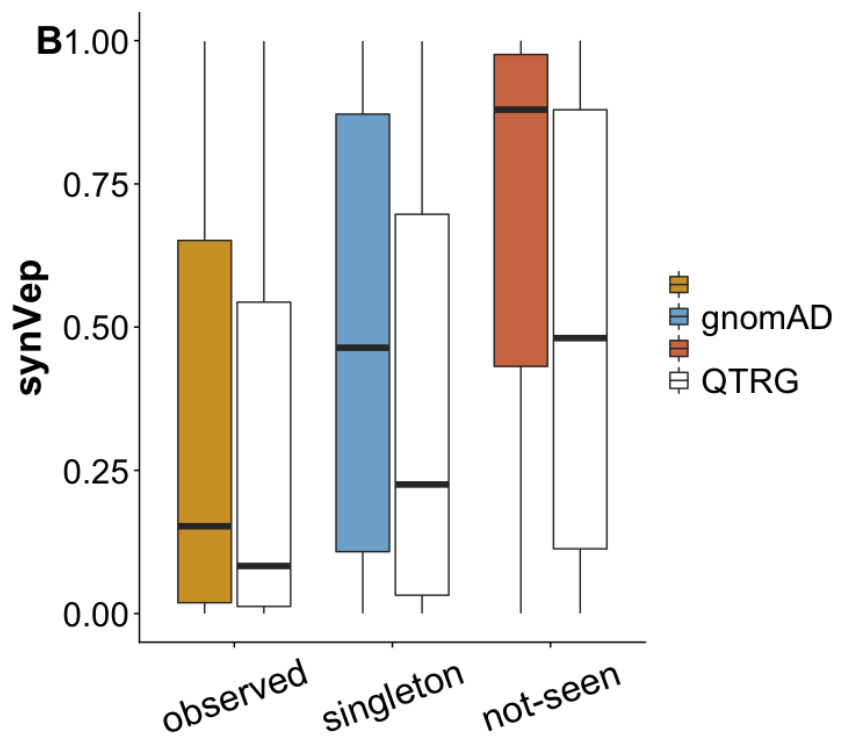

714

Figure 9. Distributions of the Qatar Genome sSNVs. In both panels, our gnomAD-based observed (orange), singleton (blue) and not-seen (dark orange) sets are highlighted. (A) represents the fraction of the QTRG sSNVs mapped our observed, singleton, not-seen, and unobservable (gray) sets. (B) synVep scores for our (gnomAD-based) variant sets, as well as the scores for QTRG sSNVs (white) mapping to the corresponding gnomAD-classes. Importantly, the synVep scores of QTRG variants that were previously classified as singletons or not-seen score much lower than other variants in the corresponding groups.

How many of the previously not-seen SSNVs are effect? New sSNVs are likely to come from clinical sequencing and could thus could often be deemed disease-associated. We expect, however, that these variants will carry little or no effect. In other words, currently not-seen no-effect observable sSNVs ( $n=14,259,180$ transcript-based and $n=5,975,076$ genomic coordinate-based) are more likely to be discovered in the future than an effect ones - even if a sample is taken from a sick individual. Recall our assumption that the not-seen set is composed of those sSNVs that carry a large effect and have been purified, as well as those that are putatively neutral and will be seen in the future if more sequencing is performed. The synVep scores of the QTRG sSNVs mapping to our not-seen set were, on average, much lower than those of the entire not-seen set (Figure 9B, average synVep score 0.49 vs. 0.88, MannWhitney $U$ test $p$-value<2.2e-16). Similarly, the median synVep score of the $2,469,205$ sSNVs not-seen according to gnomAD, but present in dbSNP (142) was lower than for the entire not-seen set ( 0.47 vs $0.88 ; p$-value $<2.2 e-16$ ). These results confirm our assumption, as these newly identified sSNVs are actually observable (not purified) and thus they are generally less likely to have large effect (and thus lower synVep scores). It may also be that the newly identified predicted effect variants (from QTRG, and 
736 other sequencing efforts in the future) are the ethnicity-differentiating, i.e. not necessarily affecting overall

737 fitness, but contributing to individual differences (as in e.g. (143)).

\section{CONCLUSION}

740 We developed synVep - a machine learning-based model for evaluating the effect of human sSNVs. Our

741 model does not use disease/deleteriousness-labeled training data. Instead, we used the signals derived

742 from observed (and corresponding generated) SSNVs from large sequencing projects. Our model

743 successfully distinguishes sSNVs with experimentally validated effect, e.g. splice-site disrupters, as well

744 as pathogenic sSNVs. Moreover, our model's predictions of cross-species variants (CSVs) correlate with

745 the evolutionary distance between human and CSV-species. While further experimental validations of

746 effect prediction are necessary, synVep's evaluation on sSNV effect will greatly contribute to our

747 understanding of biological molecular pathways in general, and of pathogenicity pathways in particular.

\section{DATA AVAILABILITY}

750 synVep webserver for online query: https://services.bromberglab.org/synvep; For local run, Python script 751 (https://bitbucket.org/bromberglab/synvep local) and prediction database

752 (https://zenodo.org/record/4763256) are also available.

\section{AUTHOR CONTRIBUTION}

$755 \mathrm{ZZ}$ and $\mathrm{YB}$ designed the study, evaluated the results, and wrote the manuscript; ZZ conducted the study; ZZ and AA 756 built the webserver.

\section{ACKNOWLEDGEMENT}

759 We thank our current and former lab members, Dr. Yannick Mahlich, Dr. Chengsheng Zhu, Dr. Maximillian Miller,

760 and Dr. Yanran Wang (all Rutgers), for all discussions and constructive suggestions. We also thank Kyle Flannery

761 (Rutgers) for the idea of testing our predictions with Qatari Genome variant data. We are also grateful to the

762 Rutgers Office of Advanced Research Computing (OARC) for making high-performance compute resources

763 available to this project, Thomas Pawlowski (Rutgers Office of Information Technology) for setting up the host of

764 synVep webserver, and to the Ensembl team for their help and feedback. Last but not least, we want to thank all 765 researchers and human subjects who made the data and tools used in this study available.

\section{FUNDING}


ZZ and YB were supported by the NIH/NIGMS grant R01 [GM115486]; AA is supported by the Astrobiology Institute grant [80NSSC18M0093]; YB was also supported by NIH grant R01 [MH115958].

\section{CONFLICT OF INTEREST}

$772 \quad$ None declared.

\section{REFERENCES}

1. Ashley, E.A. (2016) Towards precision medicine. Nature Reviews Genetics, 17, 507.

2. Visscher, P.M., Wray, N.R., Zhang, Q., Sklar, P., McCarthy, M.I., Brown, M.A. and Yang, J. (2017) 
19. Zhang, X., Li, M., Lin, H., Rao, X., Feng, W., Yang, Y., Mort, M., Cooper, D.N., Wang, Y. and Wang, Y. (2017) regSNPs-splicing: a tool for prioritizing synonymous single-nucleotide substitution. Human genetics, 136, 1279-1289.

20. Livingstone, M., Folkman, L., Yang, Y., Zhang, P., Mort, M., Cooper, D.N., Liu, Y., Stantic, B. and Zhou, Y. (2017) Investigating DNA-, RNA-, and protein-based features as a means to discriminate pathogenic synonymous variants. Human mutation, 38, 1336-1347.

823

824

21. Gelfman, S., Wang, Q., McSweeney, K.M., Ren, Z., La Carpia, F., Halvorsen, M., Schoch, K., Ratzon, F., Heinzen, E.L. and Boland, M.J. (2017) Annotating pathogenic non-coding variants in genic regions. Nature communications, 8, 236.

22. Shi, F., Yao, Y., Bin, Y., Zheng, C.-H. and Xia, J. (2019) Computational identification of deleterious synonymous variants in human genomes using a feature-based approach. BMC medical genomics, 12, 12.

23. Kircher, M., Witten, D.M., Jain, P., O'roak, B.J., Cooper, G.M. and Shendure, J. (2014) A general framework for estimating the relative pathogenicity of human genetic variants. Nature genetics, 46, 310.

24. Rentzsch, P., Witten, D., Cooper, G.M., Shendure, J. and Kircher, M. (2019) CADD: predicting the deleteriousness of variants throughout the human genome. Nucleic acids research, 47, D886D894.

25. Quang, D., Chen, Y. and Xie, X. (2014) DANN: a deep learning approach for annotating the pathogenicity of genetic variants. Bioinformatics, 31, 761-763.

26. Shihab, H.A., Rogers, M.F., Gough, J., Mort, M., Cooper, D.N., Day, I.N., Gaunt, T.R. and Campbell, C. (2015) An integrative approach to predicting the functional effects of non-coding and coding sequence variation. Bioinformatics, 31, 1536-1543.

27. Schwarz, J.M., Cooper, D.N., Schuelke, M. and Seelow, D. (2014) MutationTaster2: mutation prediction for the deep-sequencing age. Nature methods, 11, 361.

28. Stenson, P.D., Mort, M., Ball, E.V., Evans, K., Hayden, M., Heywood, S., Hussain, M., Phillips, A.D. and Cooper, D.N. (2017) The Human Gene Mutation Database: towards a comprehensive repository of inherited mutation data for medical research, genetic diagnosis and next-generation sequencing studies. Human genetics, 136, 665-677.

29. Landrum, M.J., Lee, J.M., Riley, G.R., Jang, W., Rubinstein, W.S., Church, D.M. and Maglott, D.R. (2014) ClinVar: public archive of relationships among sequence variation and human phenotype. Nucleic Acids Research, 42, D980-D985.

30. Bromberg, Y., Kahn, P.C. and Rost, B. (2013) Neutral and weakly nonneutral sequence variants may define individuality. 110, 14255-14260.

31. Pejaver, V., Babbi, G., Casadio, R., Folkman, L., Katsonis, P., Kundu, K., Lichtarge, O., Martelli, P.L., Miller, M. and Moult, J. (2019) Assessment of methods for predicting the effects of PTEN and TPMT protein variants. Human mutation, 40, 1495-1506.

32. Liu, B., Lee, W.S., Yu, P.S. and Li, X. (2002), ICML. Citeseer, Vol. 2, pp. 387-394.

33. Liu, B., Dai, Y., Li, X., Lee, W.S. and Yu, P.S. (2003), Third IEEE International Conference on Data Mining. IEEE, pp. 179-186.

34. Fusilier, D.H., Montes-y-Gómez, M., Rosso, P. and Cabrera, R.G. (2015) Detecting positive and negative deceptive opinions using PU-learning. Information processing \& management, 51, 433443.

35. Mahlich, Y., Miller, M., Zeng, Z. and Bromberg, Y. (2021) Low diversity of human variation despite mostly mild functional impact of de novo variation. Frontiers in Molecular Biosciences, 8, 74.

36. Kinsella, R.J., Kahari, A., Haider, S., Zamora, J., Proctor, G., Spudich, G., Almeida-King, J., Staines, D., Derwent, P., Kerhornou, A. et al. (2011) Ensembl BioMarts: a hub for data retrieval across taxonomic space. 2011, bar030-bar030.

37. Church, D.M., Schneider, V.A., Graves, T., Auger, K., Cunningham, F., Bouk, N., Chen, H.-C., Agarwala, R., McLaren, W.M., Ritchie, G.R.S. et al. (2011) Modernizing Reference Genome Assemblies. 9, e1001091.

38. Wang, K., Li, M. and Hakonarson, H. (2010) ANNOVAR: functional annotation of genetic variants from high-throughput sequencing data. Nucleic Acids Research, 38, e164-e164.

39. Karczewski, K.J., Francioli, L.C., Tiao, G., Cummings, B.B., Alföldi, J., Wang, Q., Collins, R.L., Laricchia, K.M., Ganna, A., Birnbaum, D.P. et al. (2019) Variation across 141,456 human exomes 
873

874

875

876

877

878

879

880

881

882

883

884

885

886

887

888

889

890

891

892

893

894

895

896

897

898

899

900

901

902

903

904

905

906

907

908

909

910

911

912

913

914

915

916

917

918

919

920

921

922

923

924

925

926

927

and genomes reveals the spectrum of loss-of-function intolerance across human protein-coding genes. bioRxiv, 531210.

40. Freedman, D., Pisani, R., Purves, R. and Adhikari, A. (2007). WW Norton \& Company New York.

41. Acock, A.C. and Stavig, G.R. (1979) A measure of association for nonparametric statistics. Social Forces, 57, 1381-1386.

42. Fisher, R.A. (1992), Breakthroughs in statistics. Springer, pp. 66-70.

43. Lonsdale, J., Thomas, J., Salvatore, M., Phillips, R., Lo, E., Shad, S., Hasz, R., Walters, G., Garcia, F. and Young, N. (2013) The genotype-tissue expression (GTEx) project. Nature genetics, 45, 580.

44. Sharp, P.M. and Li, W.-H. (1987) The codon adaptation index-a measure of directional synonymous codon usage bias, and its potential applications. Nucleic acids research, 15, 12811295.

45. Ikemura, T. (1981) Correlation between the abundance of Escherichia coli transfer RNAs and the occurrence of the respective codons in its protein genes: a proposal for a synonymous codon choice that is optimal for the E. coli translational system. Journal of molecular biology, 151, 389409.

46. Karlin, S. and Mrázek, J. (1996) What drives codon choices in human genes? Journal of molecular biology, 262, 459-472.

47. Freire-Picos, M.A., Gonzalez-Siso, M.I., Rodríguez-Belmonte, E., Rodriguez-Torres, A.M., Ramil, E. and Cerdan, M.E. (1994) Codon usage in Kluyveromyces lactis and in yeast cytochrome cencoding genes. Gene, 139, 43-49.

48. Wan, X., Xu, D. and Zhou, J. (2003) A new informatics method for measuring synonymous codon usage bias. Intelligent engineering systems through artificial neural networks Volume, 13.

49. Dos Reis, M., Wernisch, L. and Savva, R. (2003) Unexpected correlations between gene expression and codon usage bias from microarray data for the whole Escherichia coli $\mathrm{K}-12$ genome. Nucleic acids research, 31, 6976-6985.

50. Team, R.C. (2013) R: A language and environment for statistical computing.

51. Supek, F. and Vlahoviček, K. (2005) Comparison of codon usage measures and their applicability in prediction of microbial gene expressivity. BMC bioinformatics, 6, 182.

52. Cannarozzi, G., Schraudolph, N.N., Faty, M., Von Rohr, P., Friberg, M.T., Roth, A.C., Gonnet, P., Gonnet, G. and Barral, Y. (2010) A Role for Codon Order in Translation Dynamics. Cell, 141, 355-367.

53. Yevshin, I., Sharipov, R., Valeev, T., Kel, A. and Kolpakov, F. (2016) GTRD: a database of transcription factor binding sites identified by ChIP-seq experiments. Nucleic acids research, gkw951.

54. Zhao, H., Sun, Z., Wang, J., Huang, H., Kocher, J.-P. and Wang, L. (2013) CrossMap: a versatile tool for coordinate conversion between genome assemblies. Bioinformatics, 30, 1006-1007.

55. Giudice, G., Sánchez-Cabo, F., Torroja, C. and Lara-Pezzi, E. (2016) ATtRACT-a database of RNA-binding proteins and associated motifs. Database, 2016.

56. Cáceres, E.F. and Hurst, L.D. (2013) The evolution, impact and properties of exonic splice enhancers. Genome biology, 14, R143.

57. Yachdav, G., Kloppmann, E., Kajan, L., Hecht, M., Goldberg, T., Hamp, T., Hönigschmid, P., Schafferhans, A., Roos, M. and Bernhofer, M. (2014) PredictProtein-an open resource for online prediction of protein structural and functional features. Nucleic acids research, 42, W337-W343.

58. Rost, B. (1996), Methods in enzymology. Elsevier, Vol. 266, pp. 525-539.

59. Schlessinger, A., Punta, M., Yachdav, G., Kajan, L. and Rost, B. (2009) Improved disorder prediction by combination of orthogonal approaches. PloS one, 4, e4433.

60. Lorenz, R., Bernhart, S.H., Zu Siederdissen, C.H., Tafer, H., Flamm, C., Stadler, P.F. and Hofacker, I.L. (2011) ViennaRNA Package 2.0. Algorithms for Molecular Biology, 6, 26.

61. Sabarinathan, R., Tafer, H., Seemann, S.E., Hofacker, I.L., Stadler, P.F. and Gorodkin, J. (2013) RNA snp: efficient detection of local RNA secondary structure changes induced by SNP s. Human mutation, 34, 546-556.

62. LeCun, Y., Bengio, Y. and Hinton, G. (2015) Deep learning. nature, 521, 436-444.

63. Chen, T. and Guestrin, C. (2016), Proceedings of the 22nd acm sigkdd international conference on knowledge discovery and data mining. ACM, pp. 785-794. 
64. Pedregosa, F., Varoquaux, G., Gramfort, A., Michel, V., Thirion, B., Grisel, O., Blondel, M., Prettenhofer, P., Weiss, R. and Dubourg, V. (2011) Scikit-learn: Machine learning in Python. Journal of machine learning research, 12, 2825-2830.

65. Robin, X., Turck, N., Hainard, A., Tiberti, N., Lisacek, F., Sanchez, J.-C. and Müller, M. (2011) pROC: an open-source package for $\mathrm{R}$ and $\mathrm{S}+$ to analyze and compare ROC curves. $B M C$ bioinformatics, 12, 1-8.

66. Grau, J., Grosse, I. and Keilwagen, J. (2015) PRROC: computing and visualizing precision-recall and receiver operating characteristic curves in R. Bioinformatics, 31, 2595-2597.

67. Ionita-Laza, I., McCallum, K., Xu, B. and Buxbaum, J.D. (2016) A spectral approach integrating functional genomic annotations for coding and noncoding variants. Nature genetics, 48, 214.

68. Landrum, M.J. and Kattman, B.L. (2018) ClinVar at five years: Delivering on the promise. Human Mutation, 39, 1623-1630.

69. Davydov, E.V., Goode, D.L., Sirota, M., Cooper, G.M., Sidow, A. and Batzoglou, S. (2010) Identifying a high fraction of the human genome to be under selective constraint using GERP++. PLoS computational biology, 6, e1001025.

70. Cheung, R., Insigne, K.D., Yao, D., Burghard, C.P., Wang, J., Hsiao, Y.-H.E., Jones, E.M., Goodman, D.B., Xiao, X. and Kosuri, S. (2019) A multiplexed assay for exon recognition reveals that an unappreciated fraction of rare genetic variants cause large-effect splicing disruptions. Molecular cell, 73, 183-194. e188.

71. Jaganathan, K., Panagiotopoulou, S.K., McRae, J.F., Darbandi, S.F., Knowles, D., Li, Y.I., Kosmicki, J.A., Arbelaez, J., Cui, W. and Schwartz, G.B. (2019) Predicting splicing from primary sequence with deep learning. Cell, 176, 535-548. e524.

72. Rentzsch, P., Schubach, M., Shendure, J. and Kircher, M. (2021) CADD-Splice-improving genome-wide variant effect prediction using deep learning-derived splice scores. Genome medicine, 13, 1-12.

73. Kumar, S., Stecher, G., Suleski, M. and Hedges, S.B. (2017) TimeTree: a resource for timelines, timetrees, and divergence times. Molecular biology and evolution, 34, 1812-1819.

74. Zerbino, D.R., Achuthan, P., Akanni, W., Amode, M.R., Barrell, D., Bhai, J., Billis, K., Cummins, C., Gall, A. and Girón, C.G. (2017) Ensembl 2018. Nucleic acids research, 46, D754-D761.

75. Löytynoja, A. and Goldman, N. (2005) An algorithm for progressive multiple alignment of sequences with insertions. Proceedings of the National Academy of Sciences, 102, 10557-10562.

76. Havrilla, J.M., Pedersen, B.S., Layer, R.M. and Quinlan, A.R. (2019) A map of constrained coding regions in the human genome. Nature genetics, 51, 88-95.

77. Fakhro, K.A., Staudt, M.R., Ramstetter, M.D., Robay, A., Malek, J.A., Badii, R., Al-Marri, A.A.-N., Abi Khalil, C., Al-Shakaki, A. and Chidiac, O. (2016) The Qatar genome: a population-specific tool for precision medicine in the Middle East. Human genome variation, 3, 1-7.

78. Leinonen, R., Sugawara, H., Shumway, M. and Collaboration, I.N.S.D. (2010) The sequence read archive. Nucleic acids research, 39, D19-D21.

79. Dohm, J.C., Lottaz, C., Borodina, T. and Himmelbauer, H. (2008) Substantial biases in ultra-short read data sets from high-throughput DNA sequencing. Nucleic acids research, 36, e105.

80. Rieber, N., Zapatka, M., Lasitschka, B., Jones, D., Northcott, P., Hutter, B., Jäger, N., Kool, M., Taylor, M. and Lichter, P. (2013) Coverage bias and sensitivity of variant calling for four wholegenome sequencing technologies. PloS one, 8, e66621.

81. Forsdyke, D.R. (2001) Functional constraint and molecular evolution. e LS.

82. Alazami, A.M., Awad, S.M., Coskun, S., Al-Hassan, S., Hijazi, H., Abdulwahab, F.M., Poizat, C. and Alkuraya, F.S. (2015) TLE6 mutation causes the earliest known human embryonic lethality. Genome biology, 16, 1-8.

83. Shamseldin, H.E., Tulbah, M., Kurdi, W., Nemer, M., Alsahan, N., Al Mardawi, E., Khalifa, O., Hashem, A., Kurdi, A. and Babay, Z. (2015) Identification of embryonic lethal genes in humans by autozygosity mapping and exome sequencing in consanguineous families. Genome biology, 16, 116.

84. Shao, Z.-Q., Zhang, Y.-M., Feng, X.-Y., Wang, B. and Chen, J.-Q. (2012) Synonymous codon ordering: a subtle but prevalent strategy of bacteria to improve translational efficiency. PLoS One, 7, e33547. 
982 85. Tesina, P., Lessen, L.N., Buschauer, R., Cheng, J., Wu, C.C.C., Berninghausen, O., Buskirk, A.R., Becker, T., Beckmann, R. and Green, R. (2020) Molecular mechanism of translational stalling by inhibitory codon combinations and poly (A) tracts. The EMBO journal, 39, e103365.

86. Gamble, C.E., Brule, C.E., Dean, K.M., Fields, S. and Grayhack, E.J. (2016) Adjacent codons act in concert to modulate translation efficiency in yeast. Cell, 166, 679-690.

87. Boël, G., Letso, R., Neely, H., Price, W.N., Wong, K.-H., Su, M., Luff, J.D., Valecha, M., Everett, J.K. and Acton, T.B. (2016) Codon influence on protein expression in E. coli correlates with mRNA levels. Nature, 529, 358.

88. Plotkin, J.B., Robins, H. and Levine, A.J. (2004) Tissue-specific codon usage and the expression of human genes. Proceedings of the National Academy of Sciences of the United States of America, 101, 12588-12591.

89. Pop, C., Rouskin, S., Ingolia, N.T., Han, L., Phizicky, E.M., Weissman, J.S. and Koller, D. (2014) Causal signals between codon bias, mRNA structure, and the efficiency of translation and elongation. Molecular systems biology, 10, 770.

90. Qian, W., Yang, J.-R., Pearson, N.M., Maclean, C. and Zhang, J. (2012) Balanced Codon Usage Optimizes Eukaryotic Translational Efficiency. 8, e1002603.

91. Hia, F., Yang, S.F., Shichino, Y., Yoshinaga, M., Murakawa, Y., Vandenbon, A., Fukao, A., Fujiwara, T., Landthaler, M. and Natsume, T. (2019) Codon bias confers stability to human mRNA s. EMBO reports, 20, e48220.

92. Presnyak, V., Alhusaini, N., Chen, Y.-H., Martin, S., Morris, N., Kline, N., Olson, S., Weinberg, D., Baker, K.E. and Graveley, B.R. (2015) Codon optimality is a major determinant of mRNA stability. Cell, 160, 1111-1124.

93. Chamary, J.-V. and Hurst, L.D. (2005) Evidence for selection on synonymous mutations affecting stability of mRNA secondary structure in mammals. Genome biology, 6, 1-12.

94. Duan, J., Wainwright, M.S., Comeron, J.M., Saitou, N., Sanders, A.R., Gelernter, J. and Gejman, P.V. (2003) Synonymous mutations in the human dopamine receptor D2 (DRD2) affect mRNA stability and synthesis of the receptor. Human molecular genetics, 12, 205-216.

95. Jacobs, W.M. and Shakhnovich, E.I. (2017) Evidence of evolutionary selection for cotranslational folding. Proceedings of the National Academy of Sciences, 114, 11434-11439.

96. Kimchi-Sarfaty, C., Oh, J.M., Kim, I.-W., Sauna, Z.E., Calcagno, A.M., Ambudkar, S.V. and Gottesman, M.M. (2007) A" silent" polymorphism in the MDR1 gene changes substrate specificity. Science, 315, 525-528.

97. Lorenz, R., Wolfinger, M.T., Tanzer, A. and Hofacker, I.L. (2016) Predicting RNA secondary structures from sequence and probing data. Methods, 103, 86-98.

98. Hughes, A.L. (2008) Near-neutrality: the leading edge of the neutral theory of molecular evolution. Annals of the New York Academy of Sciences, 1133, 162.

99. Eyre-Walker, A. and Keightley, P.D. (2007) The distribution of fitness effects of new mutations. Nature Reviews Genetics, 8, 610-618.

100. Wall, J.D., Tang, L.F., Zerbe, B., Kvale, M.N., Kwok, P.-Y., Schaefer, C. and Risch, N. (2014) Estimating genotype error rates from high-coverage next-generation sequence data. Genome research, 24, 1734-1739.

101. Lek, M., Karczewski, K.J., Minikel, E.V., Samocha, K.E., Banks, E., Fennell, T., O'Donnell-Luria, A.H., Ware, J.S., Hill, A.J., Cummings, B.B. et al. (2016) Analysis of protein-coding genetic variation in 60,706 humans. Nature, 536, 285-291.

102. Bloom, J.S., Boocock, J., Treusch, S., Sadhu, M.J., Day, L., Oates-Barker, H. and Kruglyak, L. (2019) Rare variants contribute disproportionately to quantitative trait variation in yeast. Elife, 8, e49212.

103. Purcell, S.M., Moran, J.L., Fromer, M., Ruderfer, D., Solovieff, N., Roussos, P., O'dushlaine, C., Chambert, K., Bergen, S.E. and Kähler, A. (2014) A polygenic burden of rare disruptive mutations in schizophrenia. Nature, 506, 185-190.

104. Teng, S., Michonova-Alexova, E. and Alexov, E. (2008) Approaches and resources for prediction of the effects of non-synonymous single nucleotide polymorphism on protein function and interactions. Current pharmaceutical biotechnology, 9, 123-133.

105. Genovese, G., Fromer, M., Stahl, E.A., Ruderfer, D.M., Chambert, K., Landén, M., Moran, J.L., Purcell, S.M., Sklar, P. and Sullivan, P.F. (2016) Increased burden of ultra-rare protein-altering variants among 4,877 individuals with schizophrenia. Nature neuroscience, 19, 1433-1441. 
1061

1062

1063

1064

1065

1066

1067

1068

1069

1070

1071

1072

1073

1074

1075

1076

1077

1078

1079

1080

1081

1082

1083

1084

1085

1086

1087

1088

1089

1090

1091

106. Bobbili, D.R., Banda, P., Krüger, R. and May, P. (2020) Excess of singleton loss-of-function variants in Parkinson's disease contributes to genetic risk. Journal of Medical Genetics.

107. Zhang, D., Cheng, L., Qian, Y., Alliey-Rodriguez, N., Kelsoe, J.R., Greenwood, T., Nievergelt, C., Barrett, T.B., McKinney, R. and Schork, N. (2009) Singleton deletions throughout the genome increase risk of bipolar disorder. Molecular psychiatry, 14, 376-380.

108. Cooper, G.M. and Shendure, J. (2011) Needles in stacks of needles: finding disease-causal variants in a wealth of genomic data. Nature Reviews Genetics, 12, 628-640.

109. Eilbeck, K., Quinlan, A. and Yandell, M. (2017) Settling the score: variant prioritization and Mendelian disease. Nature Reviews Genetics, 18, 599-612.

110. MacArthur, D., Manolio, T., Dimmock, D., Rehm, H., Shendure, J., Abecasis, G., Adams, D., Altman, R., Antonarakis, S. and Ashley, E. (2014) Guidelines for investigating causality of sequence variants in human disease. Nature, 508, 469-476.

111. Torkamani, A., Wineinger, N.E. and Topol, E.J. (2018) The personal and clinical utility of polygenic risk scores. Nature Reviews Genetics, 19, 581-590.

112. Khera, A.V., Chaffin, M., Aragam, K.G., Haas, M.E., Roselli, C., Choi, S.H., Natarajan, P., Lander, E.S., Lubitz, S.A. and Ellinor, P.T. (2018) Genome-wide polygenic scores for common diseases identify individuals with risk equivalent to monogenic mutations. Nature genetics, 50, 1219-1224.

113. Wang, Y. and Bromberg, Y. (2019) Identifying mutation-driven changes in gene functionality that lead to venous thromboembolism. Human mutation, 40, 1321-1329.

114. Raimondi, D., Simm, J., Arany, A., Fariselli, P., Cleynen, I. and Moreau, Y. (2020) An interpretable low-complexity machine learning framework for robust exome-based in-silico diagnosis of Crohn's disease patients. NAR Genomics and Bioinformatics, 2, lqaa011.

115. Capra, J.A., Stolzer, M., Durand, D. and Pollard, K.S. (2013) How old is my gene? Trends in Genetics, 29, 659-668.

116. Briscoe, A.D., Gaur, C. and Kumar, S. (2004) The spectrum of human rhodopsin disease mutations through the lens of interspecific variation. Gene, 332, 107-118.

117. Waterston, R.H. and Pachter, L. (2002) Initial sequencing and comparative analysis of the mouse genome. Nature, 420, 520-562.

118. Kondrashov, A.S., Sunyaev, S. and Kondrashov, F.A. (2002) Dobzhansky-Muller incompatibilities in protein evolution. Proceedings of the National Academy of Sciences, 99, 14878-14883.

119. Subramanian, S. and Kumar, S. (2006) Evolutionary anatomies of positions and types of diseaseassociated and neutral amino acid mutations in the human genome. BMC genomics, 7, 306.

120. Li, W., Manktelow, E., von Kirchbach, J.C., Gog, J.R., Desselberger, U. and Lever, A.M. (2010) Genomic analysis of codon, sequence and structural conservation with selective biochemicalstructure mapping reveals highly conserved and dynamic structures in rotavirus RNAs with potential cis-acting functions. Nucleic acids research, 38, 7718-7735.

121. Cheng, J., Nguyen, T.Y.D., Cygan, K.J., Çelik, M.H., Fairbrother, W.G. and Gagneur, J. (2019) MMSplice: modular modeling improves the predictions of genetic variant effects on splicing. Genome biology, 20, 1-15.

122. Wang, G.-S. and Cooper, T.A. (2007) Splicing in disease: disruption of the splicing code and the decoding machinery. Nature Reviews Genetics, 8, 749-761.

123. Supek, F., Miñana, B., Valcárcel, J., Gabaldón, T. and Lehner, B. (2014) Synonymous mutations frequently act as driver mutations in human cancers. Cell, 156, 1324-1335.

124. Weile, J. and Roth, F.P. (2018) Multiplexed assays of variant effects contribute to a growing genotype-phenotype atlas. Human genetics, 137, 665-678.

125. Richards, S., Aziz, N., Bale, S., Bick, D., Das, S., Gastier-Foster, J., Grody, W.W., Hegde, M., Lyon, E. and Spector, E. (2015) Standards and guidelines for the interpretation of sequence variants: a joint consensus recommendation of the American College of Medical Genetics and Genomics and the Association for Molecular Pathology. Genetics in medicine, 17, 405-423.

126. Pertea, M., Lin, X. and Salzberg, S.L. (2001) GeneSplicer: a new computational method for splice site prediction. Nucleic acids research, 29, 1185-1190.

127. Reese, M.G., Eeckman, F.H., Kulp, D. and Haussler, D. (1997) Improved splice site detection in Genie. Journal of computational biology, 4, 311-323. 
1092

1093

1094

1095

1096

1097

1098

1099

1100

1101

1102

1103

1104

1105

1106

1107

1108

1109

1110

1111

1112

1113

1114

1115

1116

1117

1118

1119

1120

1121

1122

1123

1124

1125

1126

1127

1128

1129

1130
128. Comeron, J.M. and Kreitman, M. (1998) The correlation between synonymous and nonsynonymous substitutions in Drosophila: mutation, selection or relaxed constraints? Genetics, 150, 767-775.

129. Wyckoff, G.J., Malcom, C.M., Vallender, E.J. and Lahn, B.T. (2005) A highly unexpected strong correlation between fixation probability of nonsynonymous mutations and mutation rate. Trends in Genetics, 21, 381-385.

130. Li, J., Zhang, Z., Vang, S., Yu, J., Wong, G.K.-S. and Wang, J. (2009) Correlation between Ka/Ks and $\mathrm{Ks}$ is related to substitution model and evolutionary lineage. Journal of molecular evolution, 68, 414-423.

131. Hurst, L.D. and Pál, C. (2001) Evidence for purifying selection acting on silent sites in BRCA1. TRENDS in Genetics, 17, 62-65.

132. Orban, T.I. and Olah, E. (2001) Purifying selection on silent sites-a constraint from splicing regulation? TRENDS in Genetics, 17, 252-253.

133. Parmley, J.L. and Hurst, L.D. (2007) How Common Are Intragene Windows with K A> K S Owing to Purifying Selection on Synonymous Mutations? Journal of molecular evolution, 64, 646-655.

134. McVean, G.A. and Vieira, J. (2001) Inferring parameters of mutation, selection and demography from patterns of synonymous site evolution in Drosophila. Genetics, 157, 245-257.

135. Nielsen, R., Bauer DuMont, V.L., Hubisz, M.J. and Aquadro, C.F. (2007) Maximum likelihood estimation of ancestral codon usage bias parameters in Drosophila. Molecular Biology and Evolution, 24, 228-235.

136. Zhou, T., Gu, W. and Wilke, C.O. (2010) Detecting positive and purifying selection at synonymous sites in yeast and worm. Molecular biology and evolution, 27, 1912-1922.

137. Lawrie, D.S., Messer, P.W., Hershberg, R. and Petrov, D.A. (2013) Strong purifying selection at synonymous sites in D. melanogaster. PLoS Genet, 9, e1003527.

138. Lu, J. and Wu, C.-I. (2005) Weak selection revealed by the whole-genome comparison of the $\mathrm{X}$ chromosome and autosomes of human and chimpanzee. Proceedings of the National Academy of Sciences, 102, 4063-4067.

139. Hellmann, I., Zöllner, S., Enard, W., Ebersberger, I., Nickel, B. and Pääbo, S. (2003) Selection on human genes as revealed by comparisons to chimpanzee cDNA. Genome research, 13, 831-837.

140. Martin, A.R., Kanai, M., Kamatani, Y., Okada, Y., Neale, B.M. and Daly, M.J. (2019) Clinical use of current polygenic risk scores may exacerbate health disparities. Nature genetics, 51, 584-591.

141. Karczewski, K.J., Francioli, L.C., Tiao, G., Cummings, B.B., Alföldi, J., Wang, Q., Collins, R.L., Laricchia, K.M., Ganna, A. and Birnbaum, D.P. (2020) The mutational constraint spectrum quantified from variation in 141,456 humans. Nature, 581, 434-443.

142. Sherry, S.T., Ward, M.-H., Kholodov, M., Baker, J., Phan, L., Smigielski, E.M. and Sirotkin, K. (2001) dbSNP: the NCBI database of genetic variation. Nucleic acids research, 29, 308-311.

143. Bromberg, Y., Kahn, P.C. and Rost, B. (2013) Neutral and weakly nonneutral sequence variants may define individuality. Proceedings of the National Academy of Sciences, 110, 14255. 